\title{
The approximate inverse in action III: 3D-Doppler tomography ${ }^{\star}$
}

\author{
Andreas Rieder ${ }^{1}$, Thomas Schuster ${ }^{2}$ \\ 1 Institut für Wissenschaftliches Rechnen und Mathematische Modellbildung, Universität \\ Karlsruhe, 76128 Karlsruhe, Germany; e-mail: andreas.rieder@math.uni-karlsruhe.de \\ 2 Department of Mathematics, Tufts University, Medford, MA 02155, USA; \\ e-mail: Thomas.Schuster@tufts.edu
}

Received November 4, 2002 / Revised version received September 23, 2003 /

Published online January 8, 2004 - (C) Springer-Verlag 2004

Summary. The approximate inverse is a powerful tool for solving first kind operator equations in a stable way. Its abstract convergence and stability theory developed in our articles [SIAM J. Numer. Anal., 37, 1909-1929,2000] and [Math. Comp., 72, 1399-1415, 2003] is applied to the reconstruction problem of 3D-vector field tomography resulting in a reconstruction algorithm of filtered backprojection type. For an analytically computed reconstruction filter (reconstruction kernel) convergence with rates as well as the regularization property are established.

Mathematics Subject Classification (1991): 65J10, 65R10

\section{Introduction}

The approximate inverse is a numerical scheme to solve first kind operator equations in a stable way. In our papers $[15,16]$ we developed a convergence and regularization theory for the approximate inverse in a general setting. We further applied our abstract results to the reconstruction problem in 2D-tomography where we obtained convergence rates for the filtered backprojection algorithm.

In the present paper we demonstrate the power of the approximate inverse for reconstructing $3 \mathrm{D}$-vector fields from projection data, that is, we apply the

* The work was partially supported by a Feodor Lynen-Fellowship of the Alexander von Humboldt-Foundation.

Correspondence to: A. Rieder 
approximate inverse to the reconstruction problem in 3D-Doppler tomography.

We organized the paper as follows. In the next section we recall briefly our definition of the (fully discrete) approximate inverse and give an account on our findings in the former articles $[15,16]$. Then, we put life into the abstract concepts within the framework of vector field tomography. Especially, we compute analytically reconstruction kernels for the 3D-Doppler transform. As a result we gain convergence with rates for a filtered backprojection type reconstruction algorithm in 3D-Doppler tomography.

The approximate inverse was originally introduced by Louis and Maaß [7] as a continuous regularization technique. For further information in that direction see, e.g., $[3,5,6,8]$ by Louis and collaborators.

\section{Approximate inverse}

Suppose that we want to solve the operator equation

$$
A f=g
$$

where $A: X \rightarrow Y$ is a continuous linear operator between the real or complex infinite dimensional Hilbert spaces $X$ and $Y$. From a practical point of view the complete right hand side is not at our disposal: we can only observe finitely many moments $\Psi_{n} g \in \mathbb{K}^{n 1}$ of $g$. Here, $\Psi_{n}: Y \rightarrow \mathbb{K}^{n}$ is the linear observation operator which models the measuring or recording device. Let us assume - for the time being - that $\Psi_{n}: Y \rightarrow \mathbb{K}^{n}$ is bounded.

Instead of (2.1) we therefore have to consider the semi-discrete problem: find an $f_{n} \in X$ such that

$$
A_{n} f_{n}=g_{n}
$$

where $A_{n}=\Psi_{n} A$ and $g_{n}=\Psi_{n} g$ for a $g \in Y$. The above problem is underdetermined and we only can search for its minimum-norm solution $f_{n}^{\dagger}$ in $\mathrm{N}\left(A_{n}\right)^{\perp}$, the orthogonal complement of the null space of $A_{n}{ }^{2}$ In principal we can obtain $f_{n}^{\dagger}$ by solving the normal equation of (2.2). However, if the range of $A$ is non-closed in $Y$, that is, the generalized inverse of $A$ is unbounded, instabilities are likely to appear in computing $f_{n}^{\dagger}$ directly from the normal equation, especially under erroneous data $g_{n}$.

We stabilize the reconstruction by trying to compute moments of $f_{n}^{\dagger}$ : $\left\langle f_{n}^{\dagger}, e_{i}\right\rangle_{X}, i=1, \ldots, d$, with suitable mollifiers $e_{i} \in X$. In case $X=L^{2}(\Omega)$,

1 The field $\mathbb{K}$ denotes either $\mathbb{R}$ or $\mathbb{C}$ depending whether $X$ and $Y$ are real or complex spaces.

2 The minimum-norm solution $f_{n}^{\dagger}$ of (2.2) exist for any $g_{n} \in \mathbb{K}^{n}$ as the range of $A_{n}$ is finite dimensional and hence closed. 
$\Omega$ a domain in $\mathbb{R}^{m}$, one can think of the $e_{i}$ 's as smooth approximations to $\delta$-distributions located at points $x_{i} \in \Omega$. To the mollifiers $\left\{e_{i}\right\}$ we associate some set $\left\{b_{i}\right\} \subset X$ and approximate $f_{n}^{\dagger}$ by

$$
E_{d} f_{n}^{\dagger}:=\sum_{i=1}^{d}\left\langle f_{n}^{\dagger}, e_{i}\right\rangle_{X} b_{i} .
$$

Of course, we like to have $E_{d} f_{n}^{\dagger}$ converge to $f_{n}^{\dagger}$ as $d \rightarrow \infty$. Accordingly, we require that $E_{d}: X \rightarrow X$ satisfies the mollifier property

$$
\lim _{d \rightarrow \infty}\left\|E_{d} w-w\right\|_{X}=0 \quad \text { for any } w \in X
$$

which establishes the interplay of $\left\{e_{i}\right\}$ and $\left\{b_{i}\right\}$.

The computations of the moments is achieved by approximating $e_{i}$ in the range of $A_{n}^{*}$. To any $e_{i}$ we associate a reconstruction kernel $v_{i}^{n} \in \mathbb{K}^{n}$ by minimizing the defect $\left\|A_{n}^{*} v_{i}^{n}-e_{i}\right\|_{X}$, that is, $v_{i}^{n}$ solves the normal equation

$$
A_{n} A_{n}^{*} v_{i}^{n}=A_{n} e_{i} .
$$

The above equation for $v_{i}^{n}$ is independent of the data $g_{n}$, therefore, the kernels can be computed in advance without affection of noise from measurement errors. We call $\left(e_{i}, v_{i}^{n}\right)$ a mollifier/reconstruction kernel pair for $A_{n}$ because the following lemma holds true.

Lemma 2.1 Assume that $A \in \mathcal{L}(X, Y)$ and $A_{n} \in \mathcal{L}\left(X, \mathbb{K}^{n}\right)$. Further, let either $g$ be in $\mathrm{R}(A)$, the range of $A$, or $v_{i}^{n}$, a solution of $(2.5)$, be in $\mathrm{N}\left(A_{n}^{*}\right)^{\perp}$. Then,

$$
\left\langle f_{n}^{\dagger}, e_{i}\right\rangle_{X}=\left\langle g_{n}, v_{i}^{n}\right\rangle_{\mathbb{K}^{n}} .
$$

Proof. The reconstruction kernel satisfies $A_{n}^{*} v_{i}^{n}=P_{\mathrm{N}\left(A_{n}\right)} \perp e_{i}$. ${ }^{3}$ Hence,

$$
\left\langle f_{n}^{\dagger}, e_{i}\right\rangle_{X}=\left\langle f_{n}^{\dagger}, P_{\mathrm{N}\left(A_{n}\right)} e_{i}\right\rangle_{X}=\left\langle A_{n} f_{n}^{\dagger}, v_{i}^{n}\right\rangle_{\mathbb{K}^{n}}=\left\langle P_{\mathrm{R}\left(A_{n}\right)} g_{n}, v_{i}^{n}\right\rangle_{\mathbb{K}^{n}} .
$$

In case $g \in \mathrm{R}(A)$ we have $g_{n}=\Psi_{n} g=\Psi_{n} A u$ for an $u \in X$, so that $P_{\mathrm{R}\left(A_{n}\right)} g_{n}=g_{n}$. If $v_{i}^{n} \in \mathrm{N}\left(A_{n}^{*}\right)^{\perp}=\mathrm{R}\left(A_{n}\right)$ then $P_{\mathrm{R}\left(A_{n}\right)} v_{i}^{n}=v_{i}^{n}$. In both instances we obtain $\left\langle P_{\mathrm{R}\left(A_{n}\right)} g_{n}, v_{i}^{n}\right\rangle_{\mathbb{K}^{n}}=\left\langle g_{n}, v_{i}^{n}\right\rangle_{\mathbb{K}^{n}}$.

Finally we define the approximate inverse $\widetilde{A}_{n, d}: \mathbb{K}^{n} \rightarrow X$ of $A_{n}$ by

$$
\widetilde{A}_{n, d} w:=\sum_{i=1}^{d}\left\langle w, v_{i}^{n}\right\rangle_{\mathbb{K}^{n}} b_{i} .
$$

${ }^{3}$ Let $M$ be a closed subspace of the Hilbert space $Z$. By $P_{M} \in \mathcal{L}(Z)$ we denote the orthogonal projector onto $M$. 
Please note that the computation of $\widetilde{A}_{n, d} w$ reduces to an evaluation of $d$ inner products of length $n$. By Lemma 2.1 and (2.4),

$$
\widetilde{A}_{n, d} g_{n}=E_{d} f_{n}^{\dagger} \stackrel{d \rightarrow \infty}{\longrightarrow} f_{n}^{\dagger}=P_{\mathrm{N}\left(A_{n}\right)^{\perp}} f
$$

which justifies the notation approximate inverse (the equality on the right hand side holds true if $g_{n}=A_{n} f$ ).

For several reasons we wish to avoid solving the normal equation (2.5): $A_{n} A_{n}^{*}$ may be a high dimensional, densely populated, and ill-conditioned matrix; increasing $n$ calls for complete new computation of the kernels; invariances of $A$ and $A^{*}$, which are used by Louis [5] to improve the efficiency of the continuous approximate inverse (more details below in Lemma 2.3), are in general not transmitted to $A_{n}$ or $A_{n}^{*}$, respectively. Furthermore, it may even happen, for instance, if $A$ is the Radon or Doppler transform, that $A_{n}$ : $\mathrm{D}\left(A_{n}\right) \subset X \rightarrow \mathbb{K}^{n}$ is unbounded and $A_{n}^{*}$ does not exist. Then, the reconstruction kernels are not meaningfully defined by (2.5).

To overcome the problems with the above described approach we go back to the continuous situation. First, we define precisely the observation operator covering the situation where $A_{n}$ becomes unbounded. To this end $A$ is assumed to have the following mapping property

$$
A: X_{1} \rightarrow Y_{1} \text { is continuous }
$$

where $X_{1}$ and $Y_{1}$ are Banach spaces such that the embeddings $X_{1} \hookrightarrow X$ as well as $Y_{1} \hookrightarrow Y$ are continuous, injective, and dense. One can consider $X_{1}$ and $Y_{1}$ subspaces of $X$ and $Y$, respectively, which contain "smooth" elements. For instance, if $X$ and $Y$ are $L^{2}$-spaces, $X_{1}$ and $Y_{1}$ could be Sobolev spaces.

Now we define the observation operator $\Psi_{n}: Y_{1} \rightarrow \mathbb{K}^{n}$. Given $n$ functionals $\left\{\psi_{n, k}\right\}_{1 \leq k \leq n}$ in $Y_{1}^{\prime}$, the dual to $Y_{1}$, let

$$
\left(\Psi_{n} v\right)_{k}:=\left\langle\psi_{n, k}, v\right\rangle_{Y_{1}^{\prime} \times Y_{1}}, \quad k=1, \ldots, n,
$$

where $\langle\cdot, \cdot\rangle_{Y_{1}^{\prime} \times Y_{1}}$ is the duality pairing on $Y_{1}^{\prime} \times Y_{1}$.

The semi-discrete operator $A_{n}:=\Psi_{n} A: \mathrm{D}\left(A_{n}\right) \subset X \rightarrow \mathbb{K}^{n}$ with domain of definition $\mathrm{D}\left(A_{n}\right)=X_{1}$ might be bounded or unbounded. The former is the case if $X=X_{1}$ (topologically). Here $A_{n}^{*}$ exists, hence, the reconstruction kernels are well defined by (2.5). Typical examples are integral operators with smooth (integral) kernels.

Since $\mathrm{R}\left(A^{*}\right)$ is dense in $\mathrm{N}(A)^{\perp}$ we find, to any $\varepsilon_{i}>0$, an $v_{i} \in Y_{1}$ ( $Y_{1}$ is dense in $Y$ ) such that

$$
\left\|P_{\mathrm{N}(A) \perp} e_{i}-A^{*} v_{i}\right\|_{X} \leq \varepsilon_{i}, \quad i=1, \ldots, d .
$$

In [15, Sect. 3.2] we demonstrated how to obtain $v_{i}$ numerically from $e_{i}$ knowing a singular value decomposition of $A$. For $A$ being the Radon transform, 
pairs $\left(e_{i}, v_{i}\right)$ are explicitely known satisfying (2.9) with $\varepsilon_{i}=0$, see, e.g., [12, 14]. Later in this paper (Sect. 4) we analytically compute pairs $\left(e_{i}, v_{i}\right)$ for the 3D-Doppler transform where also $\varepsilon_{i}=0$.

With the $v_{i}$ 's we define approximate reconstruction kernels by

$$
v_{i}^{n}:=G_{n} \Psi_{n} v_{i}, \quad i=1, \ldots, d .
$$

The $n \times n$-matrix $G_{n}$ is the Gramian relative to a family $\left\{\varphi_{k}\right\}_{1 \leq k \leq n}$ in $Y$ which is closely connected to $\Psi_{n}$ by the operator $\Pi_{n}: Y_{1} \rightarrow Y$,

$$
\Pi_{n} v:=\sum_{k=1}^{n}\left(\Psi_{n} v\right)_{k} \varphi_{k}=\sum_{k=1}^{n}\left\langle\psi_{n, k}, v\right\rangle_{Y_{1}^{\prime} \times Y_{1}} \varphi_{k}
$$

The operator $\Pi_{n}$ is required to satisfy the approximation property (2.12): let there be a sequence $\left\{\rho_{n}\right\} \subset[0,1]$ converging monotonically to zero such that

$$
\left\|v-\Pi_{n} v\right\|_{Y} \lesssim \rho_{n}\|v\|_{Y_{1}} \quad \text { for all } v \in Y_{1} \text { as } n \rightarrow \infty .
$$

Our notation $A \lesssim B$ indicates the existence of a generic constant $c>0$ such that $A \leq c B$. The constant $c$ will not depend on the arguments of $A$ and $B$. This means that the constant involved in (2.12) does not depend on $n$ and $v$.

Furthermore, $\Pi_{n}$ is assumed to be uniformly bounded in $n$,

$$
\left\|\Pi_{n}\right\|_{Y_{1} \rightarrow Y} \lesssim 1 \quad \text { as } n \rightarrow \infty,
$$

Now we have all ingredients to formulate the convergence result. For the stability result see [16, Sect. 4.2].

Theorem 2.2 Let $A, E_{d}, \Psi_{n}$, and $\Pi_{n}$ be as specified in this section. Additionally, let the families $\left\{b_{i}\right\}_{1 \leq i \leq d} \subset X$ and $\left\{\varphi_{k}\right\}_{1 \leq k \leq n} \subset Y$ be Riesz systems. ${ }^{4}$ Let the triplets $\left\{\left(e_{i}, v_{i}, b_{i}\right)\right\}_{1 \leq i \leq d} \subset X \times Y_{1} \times X$ satisfy (2.4) and (2.9). If $f \in X_{1}$ then

$$
\begin{aligned}
\left\|\widetilde{A}_{n, d} A_{n} f-P_{\mathrm{N}(A)^{\perp}} f\right\|_{X} \lesssim & \left\|\left(I-E_{d}\right) P_{\mathrm{N}(A)^{\perp}} f\right\|_{X} \\
& +\left(\frac{1}{d} \sum_{i=1}^{d}\left(\rho_{n}^{2}\left\|v_{i}\right\|_{Y_{1}}^{2}+\varepsilon_{i}^{2}\right)\right)^{1 / 2}\|f\|_{X_{1}} .
\end{aligned}
$$

Provided $d^{-1} \sum_{i=1}^{d} \varepsilon_{i}^{2} \rightarrow 0$ as $d \rightarrow \infty$ and $\rho_{n}^{2} d^{-1} \sum_{i=1}^{d}\left\|v_{i}\right\|_{Y_{1}}^{2} \rightarrow 0$ as $n, d \rightarrow \infty$, we have convergence:

$$
\lim _{\substack{n \rightarrow \infty \\ d \rightarrow \infty}}\left\|\widetilde{A}_{n, d} A_{n} f-P_{\mathrm{N}(A) \perp} f\right\|_{X}=0 .
$$

${ }^{4}$ A family $\left\{z_{j}\right\}_{1 \leq j \leq m}$ of a Hilbert space $Z$ is called Riesz system iff

$$
m^{-1}\|w\|_{\mathbb{K}^{m}}^{2} \lesssim\left\|\sum_{j=1}^{m} w_{j} z_{j}\right\|_{Z}^{2} \lesssim m^{-1}\|w\|_{\mathbb{K}^{m}}^{2} \quad \text { for all } w \in \mathbb{K}^{m}
$$


Proof. In [16] we proved a less general version of Theorem 2.2: we restricted ourselves to injective operators, that is, $P_{\mathrm{N}(A)^{\perp}}=I$. However, the proof of Theorem 4.1 from [16] carries over to the present situation because

$$
\left|\left\langle P_{\mathrm{N}(A)^{\perp}} f, e_{i}\right\rangle_{X}-\left\langle f, A^{*} v_{i}\right\rangle_{X}\right|=\left|\left\langle f, P_{\mathrm{N}(A)^{\perp}} e_{i}-A^{*} v_{i}\right\rangle_{X}\right| \leq \varepsilon_{i}\|f\|_{X}
$$

which is all we need.

Louis [5] observed that invariances of $A$ and $A^{*}$ can be used to generate a new mollifier/reconstruction kernel pair from another one. In Lemma 2.3 below we present such a technique well suited for our version of the approximate inverse. Moreover, we only require an invariance of $A^{*}$. In this respect Lemma 2.3 is an abstract modification of Theorem 3.1 from [11]. As a practical consequence we only need to find one single pair $(e, v) \in X \times Y$ fulfilling (2.9), see Sect. 3.2.

Lemma 2.3 Let $A \in \mathcal{L}(X, Y), T \in \mathcal{L}(X)$, and $S \in \mathcal{L}(Y)$ satisfying $T A^{*}=$ $A^{*} S$. Further, let $S$ have a dense range and let $T$ be a multiple of an isometry: there is a $\tau>0$ such that $\|T u\|_{X}=\tau\|u\|_{X}$ for all $u \in X$.

$$
\text { If }\left\|P_{\mathrm{N}(A) \perp} e-A^{*} v\right\|_{X} \leq \varepsilon \text { for a pair }(e, v) \in X \times Y \text { then also }
$$

$$
\left\|P_{\mathrm{N}(A) \perp} T e-A^{*} S v\right\|_{X} \leq \tau \varepsilon .
$$

Proof. We claim that $P_{\mathrm{N}(A)^{\perp}} T=T P_{\mathrm{N}(A)^{\perp}}$. Having verified our claim we are done since

$$
\left\|P_{\mathrm{N}(A) \perp} T e-A^{*} S v\right\|_{X}=\left\|T\left(P_{\mathrm{N}(A)^{\perp}} e-A^{*} v\right)\right\|_{X} \leq \tau \varepsilon .
$$

Now we will establish the inclusions $T \mathrm{~N}(A)^{\perp} \subset \mathrm{N}(A)^{\perp}$ and $T \mathrm{~N}(A) \subset$ $\mathrm{N}(A)$.

To any $w \in \mathrm{N}(A)^{\perp}=\overline{\mathrm{R}\left(A^{*}\right)}$ there exist a sequence $\left\{z_{i}\right\}$ in $Y$ with $w=\lim _{i \rightarrow \infty} A^{*} z_{i}$. By the invariance we have $A^{*} S z_{i}=T A^{*} z_{i}$ which gives $\lim _{i \rightarrow \infty} A^{*} S z_{i}=T w$. Hence, $T w \in \overline{\mathrm{R}\left(A^{*}\right)}=\mathrm{N}(A)^{\perp}$ and the first inclusion is shown.

To prove the second inclusion we note that $\tau^{2} A=S^{*} A T$ which follows from $T A^{*}=A^{*} S$ when considering $T^{*} T=\tau^{2} I_{X}$ (the latter relation holds true since $T / \tau$ is an isometry, see, e.g., Weidmann [19, Theorem 4.34]). By assumption $S^{*}$ possesses a trivial null space, thus, we have $T \mathrm{~N}(A) \subset \mathrm{N}(A)$.

Finally, for any $u \in X$,

$$
P_{\mathrm{N}(A)^{\perp}} T u=P_{\mathrm{N}(A)^{\perp}} \underbrace{T P_{\mathrm{N}(A)^{\perp}} u}_{\in \mathrm{N}(A)^{\perp}}+P_{\mathrm{N}(A)^{\perp}} \underbrace{T P_{\mathrm{N}(A)} u}_{\in \mathrm{N}(A)}=T P_{\mathrm{N}(A)^{\perp}} u
$$

and our claim from the beginning is true. 


\section{Convergence of filtered backprojection type algorithm for 3D vector tomography}

Vector tomography entails the reconstruction of a vector field from line integrals over certain components of the field. There exists a wide area of applications for vector tomography ranging from medical imaging (cancer diagnosis from blood flow), structural mechanics, oceanography, photoelasticity to plasma physics, see Sparr and Stråhlén [18] for an overview.

In the present section we apply our abstract convergence results from the former sections to the reconstruction problem in $3 \mathrm{D}$ vector tomography.

\subsection{Doppler transform: definition and smoothing property}

The mathematical model for vector tomography is the Doppler transform. Here we present a convenient parameterization of this mapping and recall some of its properties which we will need later. The material is taken from [10] and [11].

We start with providing some notation. First we introduce the unit vectors $w_{1}=(0,0,1)^{t}, w_{2}=(1,0,0)^{t}$, and $w_{3}=(0,1,0)^{t}$ permuting the canonical unit vectors. With each of these vectors we associate embeddings $\mathcal{P}_{j}: \mathbb{R}^{2} \rightarrow$ $w_{j}^{\perp}, j=1,2,3$, by $\mathcal{P}_{1}\left(x_{1}, x_{2}\right)=\left(x_{1}, x_{2}, 0\right)^{t}, \mathcal{P}_{2}\left(x_{1}, x_{2}\right)=\left(0, x_{1}, x_{2}\right)^{t}$, and $\mathcal{P}_{3}\left(x_{1}, x_{2}\right)=\left(x_{1}, 0, x_{2}\right)^{t}$.

In the sequel we will only consider lines being parallel to one of the three planes $w_{j}^{\perp}, j=1,2,3$. A line $L_{j}$ parallel to $w_{j}^{\perp}$ is determined uniquely by three parameters: a direction (angle) $\omega(\vartheta)=(\cos \vartheta, \sin \vartheta)^{t}$, the distance $s \in \mathbb{R}$ to the $w_{j}$-axis, and the distance $a \in \mathbb{R}$ to the origin. Hence,

$$
L_{j}=L_{j}(\vartheta, s, a)=\left\{x \in \mathbb{R}^{3} \mid\left\langle x, \mathcal{P}_{j} \omega(\vartheta)\right\rangle=s,\left\langle x, w_{j}\right\rangle=a\right\} .
$$

Let $\Omega$ be the open unit ball in $\mathbb{R}^{3}$ centered about the origin. The $3 D$ Doppler transform

$$
\mathbf{D}=\left(\mathbf{D}_{1}, \mathbf{D}_{2}, \mathbf{D}_{3}\right)^{t}: L^{2}(\Omega)^{3} \rightarrow L^{2}(Q)^{3}, \quad Q:=[0,2 \pi] \times[-1,1]^{2},
$$

is defined by

$$
\mathbf{D}_{j} f(\vartheta, s, a)=\int_{L_{j}(\vartheta, s, a) \cap \Omega}\left\langle w_{j} \times \mathcal{P}_{j} \omega(\vartheta), f(x)\right\rangle \mathrm{d} \sigma(x), \quad j=1,2,3 .
$$

Observe that $\mathbf{D}_{2}$ and $\mathbf{D}_{3}$ are not obtained from $\mathbf{D}_{1}$ by a mere cyclic shift of $\{1,2,3\}$ : While $\mathcal{P}_{1}$ and $\mathcal{P}_{2}$ can be obtained from each other by a cyclic shift, $\mathcal{P}_{3}$ is not a cyclic shift, neither form $\mathcal{P}_{1}$ nor from $\mathcal{P}_{2}$. Thus, $\mathbf{D}_{j}$ integrates two different components of the vector field $f$ over lines located in different planes. Our definition of the $\mathbf{D}_{j}$ 's corresponds exactly to the measurement 
geometry suggested by JUHLIN [4], see also NORTON [13]. Since we intend to invert approximately a semi-discrete version of $\mathbf{D}$, see Section 3.2 below, we cannot confine to $\mathbf{D}_{1}$ only but have to use a setting involving all components of $\mathbf{D}$.

The Doppler transform satisfies a smoothing property which we formulate in Theorem 3.1 below. To this end we need further notation. We define anisotropic Sobolev spaces $\mathcal{X}_{j}^{\alpha, \beta}, \alpha, \beta \geq 0$, to be the closure of $\mathcal{C}_{0}^{\infty}(\Omega)$, the space of $\mathcal{C}^{\infty}$-functions compactly supported in $\Omega$, with respect to the norm

$\|v\|_{\mathcal{X}_{j}^{\alpha, \beta}}:=\left(\int_{\mathbb{R}^{3}}\left(1+\xi_{1}^{2}+\xi_{2}^{2}\right)^{\alpha}\left(1+\xi_{3}^{2}\right)^{\beta}\left|\widehat{v}\left(\mathcal{P}_{j}\left(\xi_{1}, \xi_{2}\right)+\xi_{3} w_{j}\right)\right|^{2} \mathrm{~d} \xi\right)^{1 / 2}$,

$j=1,2,3$. Above, $\widehat{v}$ denotes the Fourier transform of $v$. Please observe that $\mathcal{P}_{j}\left(\xi_{1}, \xi_{2}\right)+\xi_{3} w_{j}$ is only a permutation of the entries of $\xi=\left(\xi_{1}, \xi_{2}, \xi_{3}\right)^{t}$. For example,

$$
\|v\|_{\mathcal{X}_{3}^{\alpha, \beta}}=\left(\int_{\mathbb{R}^{3}}\left(1+\xi_{1}^{2}+\xi_{3}^{2}\right)^{\alpha}\left(1+\xi_{2}^{2}\right)^{\beta}|\widehat{v}(\xi)|^{2} \mathrm{~d} \xi\right)^{1 / 2} .
$$

Further, we introduce $H^{\alpha}(G)$, the $L^{2}$-Sobolev space of order $\alpha \geq 0$ over a domain $G \subset \mathbb{R}^{n}$, and its subspace $H_{0}^{\alpha}(G)$ containing all elements of $H^{\alpha}(G)$ which vanish at the boundary of $G$. For a detailed definition see, e.g., Wloka [20].

Theorem 3.1 The Doppler transform $\mathbf{D}$ is a bounded linear mapping from the Cartesian product space $\left(\mathcal{X}_{1}^{\alpha, \beta} \cap \mathcal{X}_{3}^{\alpha, \beta}\right) \times\left(\mathcal{X}_{1}^{\alpha, \beta} \cap \mathcal{X}_{2}^{\alpha, \beta}\right) \times\left(\mathcal{X}_{2}^{\alpha, \beta} \cap \mathcal{X}_{3}^{\alpha, \beta}\right)$ to the tensor product space $\left(H^{\alpha+1 / 2}(Z) \widehat{\otimes} H_{0}^{\beta}(-1,1)\right)^{3}$ where $\left.Z=\right] 0,2 \pi[$ $\times]-1,1[$.

More precisely, we have the continuity estimates

(3.1a) $\left\|\mathbf{D}_{j} f\right\|_{H^{\alpha+1 / 2}(Z) \widehat{\otimes} H_{0}^{\beta}(-1,1)} \lesssim\left\|f_{j}\right\|_{\mathcal{X}_{j}^{\alpha, \beta}}+\left\|f_{j+1}\right\|_{\mathcal{X}_{j}^{\alpha, \beta},}, j=1,2$,

(3.1b) $\left\|\mathbf{D}_{3} f\right\|_{H^{\alpha+1 / 2}(Z) \widehat{\otimes} H_{0}^{\beta}(-1,1)} \lesssim\left\|f_{1}\right\|_{\mathcal{X}_{3}^{\alpha, \beta}}+\left\|f_{3}\right\|_{\mathcal{X}_{3}^{\alpha, \beta}}$.

Proof. The proof of Theorem 2.10 from [10] actually verifies (3.1).

Basically, the above theorem tells us that $\mathbf{D} f$ is smoother in its first two arguments than $f$ by $1 / 2$ measured in an appropriate Sobolev scale.

Please note that the components of the Doppler transform $\mathbf{D}: L^{2}(\Omega)^{3} \rightarrow$ $L^{2}(Q)^{3}$ will play the role of the operator $A: X \rightarrow Y$ from our abstract setting. Hence, (3.1) corresponds to the mapping property (2.7) with $X_{1}$ and $Y_{1}$ being the spaces

$$
\boldsymbol{X}^{\alpha, \beta}:=\left(\mathcal{X}_{1}^{\alpha, \beta} \cap \mathcal{X}_{3}^{\alpha, \beta}\right) \times\left(\mathcal{X}_{1}^{\alpha, \beta} \cap \mathcal{X}_{2}^{\alpha, \beta}\right) \times\left(\mathcal{X}_{2}^{\alpha, \beta} \cap \mathcal{X}_{3}^{\alpha, \beta}\right)
$$


(note that $\boldsymbol{X}^{0,0}=L^{2}(\Omega)^{3}$ ) and

$$
\mathcal{Y}^{\alpha, \beta}:=H^{\alpha+1 / 2}(Z) \widehat{\otimes} H_{0}^{\beta}(-1,1),
$$

respectively.

\subsection{Approximate inverse for the Doppler transform}

In this subsection we provide all ingredients necessary to apply the approximate inverse to the reconstruction of vector fields from discrete Doppler data. These ingredients are mollifiers and reconstruction kernels, the observation operator $\Psi_{n}$, see (2.8), the interpolation-like operator $\Pi_{n}$, see (2.11), and the mollifier operator $E_{d}$, see (2.3).

First we introduce observation operators. Let $\ell \in\{1,2\}$ (with $\ell$ we distinguish two different scenarios) and define

$$
\begin{aligned}
\vartheta_{j} & =j h_{\vartheta}, & h_{\vartheta} & =2 \pi / p, & j & =0, \ldots, p_{\ell}, \\
s_{i} & =i h_{s}, & h_{s} & =1 / q, & i & =-q, \ldots, q_{\ell}, \\
a_{k} & =k h_{a}, & h_{a} & =1 / r, & k & =-r, \ldots, r_{\ell},
\end{aligned}
$$

where $(p, q, r \in \mathbb{N})$

$$
\begin{array}{lll}
p_{1}=p-1, & q_{1}=q-1, & r_{1}=r-1, \\
p_{2}=p, & q_{2}=q, & r_{2}=r .
\end{array}
$$

If $\alpha>1 / 2$ then point evaluations are stable operations on $H^{\alpha+1 / 2}(Z)$ as well as $H_{0}^{\alpha}(-1,1)$. Therefore, we define the bounded operators

$$
\begin{aligned}
\Psi_{p, q}^{(\ell)}: H^{\alpha+1 / 2}(Z) \rightarrow \mathbb{R}^{d_{\ell}} \quad \text { by } \quad\left(\Psi_{p, q}^{(\ell)} y\right)_{i, j}:=y\left(\vartheta_{j}, s_{i}\right), \\
d_{\ell}=\left(p_{\ell}+1\right)\left(q+q_{\ell}+1\right), \text { and }(\beta>1 / 2) \\
\Psi_{r}^{(\ell)}: H_{0}^{\beta}(-1,1) \rightarrow \mathbb{R}^{m_{\ell}} \quad \text { by } \quad\left(\Psi_{r}^{(\ell)} y\right)_{k}:=y\left(a_{k}\right),
\end{aligned}
$$

$m_{\ell}=r+r_{\ell}+1$. The tensor product of both latter point evaluations acts continuously on $\mathcal{Y}^{\alpha, \beta}$ with range $\mathbb{R}^{n_{\ell}}$ where $n_{\ell}=d_{\ell} \cdot m_{\ell}$ :

$$
\begin{aligned}
\Psi_{p, q, r}^{(\ell)}:= & \Psi_{p, q}^{(\ell)} \otimes \Psi_{r}^{(\ell)}: \mathcal{Y}^{\alpha, \beta} \longrightarrow \mathbb{R}^{d_{\ell}} \otimes \mathbb{R}^{m_{\ell}}=\mathbb{R}^{n_{\ell}} \\
& \text { is bounded for } \alpha, \beta>1 / 2 .
\end{aligned}
$$

We have

$$
n_{1}=4 p q r \quad \text { and } \quad n_{2}=(p+1)(2 q+1)(2 r+1) .
$$


By $\Psi_{p, q, r}^{(\ell)} \mathbf{D}$ we understand $\left(\Psi_{p, q, r}^{(\ell)} \mathbf{D}_{1}, \Psi_{p, q, r}^{(\ell)} \mathbf{D}_{2}, \Psi_{p, q, r}^{(\ell)} \mathbf{D}_{3}\right)^{t}$ which is well defined since $\mathbf{D}_{j}\left(\boldsymbol{X}^{\alpha}\right) \subset \mathcal{Y}^{\alpha, \beta}$, see Theorem 3.1. The reconstruction problem in $3 \mathrm{D}$ vector tomography now reads $(\alpha, \beta>1 / 2)$ :

$$
\text { given } g_{p, q, r} \in \mathbb{R}^{3 n_{\ell}} \text { find } f \in \boldsymbol{X}^{\alpha, \beta} \text { such that } \Psi_{p, q, r}^{(\ell)} \mathbf{D} f=g_{p, q, r} .
$$

For applying the approximate inverse to the reconstruction problem we consider $\Psi_{p, q, r}^{(\ell)} \mathbf{D}$ in the natural $L^{2}$-topology in which $\mathbf{D}$ is bounded. Unfortunately the $L^{2}$-boundedness of $\mathbf{D}$ does not carry over to the semi-discrete Doppler transform.

Lemma 3.2 The semi-discrete Doppler transform

$$
\Psi_{p, q, r}^{(\ell)} \mathbf{D}: \boldsymbol{X}^{\alpha, \beta} \subset L^{2}(\Omega)^{3} \rightarrow \mathbb{R}^{3 n_{\ell}}
$$

is unbounded for any $\alpha, \beta>1 / 2$. Here, $\boldsymbol{X}^{\alpha, \beta}$ is considered as the domain of definition of $\Psi_{p, q, r}^{(\ell)} \mathbf{D}$ in the $L^{2}$-topology.

In other words: The semi-discrete Doppler transform $\Psi_{p, q, r}^{(\ell)} \mathbf{D}$ has no bounded extension onto $L^{2}(\Omega)^{3}$.

Proof. The proof of Theorem 5.1 in [15] can be adapted to the present situation.

We construct the operator $\Pi_{p, q, r}^{(\ell)}$ related to $\Psi_{p, q, r}^{(\ell)}$ by the help of the tensor product spline spaces $V_{p, q, r}^{(\ell)}=S_{\vartheta}^{(\ell)} \otimes S_{s}^{(\ell)} \otimes S_{a}^{(\ell)} \subset L^{2}(Q)$. The univariate spaces $S_{\vartheta}^{(\ell)}, S_{s}^{(\ell)}$, and $S_{a}^{(\ell)}$ are the piecewise constant $(\ell=1)$ or linear $(\ell=2)$ spline spaces with respect to the knot sequences $\left\{\vartheta_{j}\right\},\left\{s_{i}\right\}$, and $\left\{a_{k}\right\}$, respectively. We equip $V_{p, q, r}^{(\ell)}$ with the tensor product B-spline basis

$$
\left\{B_{p, j}^{(\ell)} \otimes B_{q, i}^{(\ell)} \otimes B_{r, k}^{(\ell)} \mid j, i, k \text { as in (3.2) }\right\} .
$$

For instance we have that

$$
B_{p, j}^{(1)}=\chi_{\left[\vartheta_{j}, \vartheta_{j+1}[\right.} \quad \text { and } \quad B_{r, k}^{(2)}\left(a_{t}\right)=\left\{\begin{array}{ccc}
1 & : & t=k \\
0 & : & \text { otherwise }
\end{array}\right.
$$

where $\chi_{I}$ is the indicator function of the interval $I$. The other basis functions are defined accordingly. The interpolation operator $\Pi_{p, q, r}^{(\ell)}: \mathcal{Y}^{\alpha, \beta} \rightarrow V_{p, q, r}$, $\alpha, \beta>1 / 2$,

$$
\Pi_{p, q, r}^{(\ell)} y:=\sum_{j=0}^{p_{\ell}} \sum_{i=-q}^{q_{\ell}} \sum_{k=-r}^{r_{\ell}}\left(\Psi_{p, q, r}^{(\ell)} y\right)_{i, j, k} B_{p, j}^{(\ell)} \otimes B_{q, i}^{(\ell)} \otimes B_{r, k}^{(\ell)},
$$

satisfies the uniform boundedness

$$
\left\|\Pi_{p, q, r}^{(\ell)} y\right\|_{L^{2}(Q)} \lesssim\|y\|_{\mathcal{Y}^{\alpha, \beta}}
$$


as well as the approximation property

$$
\left\|\Pi_{p, q, r}^{(\ell)} y-y\right\|_{L^{2}(Q)} \lesssim \rho\|y\|_{\mathcal{Y}^{\alpha, \beta}}
$$

where

$$
\rho=\rho\left(\ell, \alpha, \beta, h_{\vartheta}, h_{s}, h_{a}\right):=\max \left\{h_{\vartheta}, h_{s}\right\}^{\min \{\alpha+1 / 2, \ell\}}+h_{a}^{\min \{\beta, \ell\}}
$$

For both latter estimates see Appendix A.

We define the mollifier operator $\mathbf{E}_{d}: L^{2}(\Omega)^{3} \rightarrow L^{2}(\Omega)^{3}$ by, $j=1,2,3$,

$$
\begin{aligned}
\left(\mathbf{E}_{d}\right)_{j} f(x) & :=\sum_{k \in \mathbb{Z}^{3}}\left\langle f_{j}, e_{d, k}^{j}\right\rangle_{L^{2}(\Omega)} B(d x-k) \\
& =\sum_{k \in \mathbb{Z}^{3}}\left\langle f, e_{d, k}^{j} \delta_{j}\right\rangle_{L^{2}(\Omega)^{3}} B(d x-k)
\end{aligned}
$$

where $B$ is the tensor product linear B-spline: $B=b \otimes b \otimes b$ with $b$ being the convolution of the indicator function of $[-1 / 2,1 / 2]$ with itself and $\delta_{j}$ is the $j$-th canonical unit vector in $\mathbb{R}^{3}$. The mollifiers used in defining $\mathbf{E}_{d}$ are scaled and translated versions of the mollifiers $e^{j}=e_{0,0}^{j}$ :

$$
e_{d, k}^{j}(x)=\mathcal{T}_{j}^{d, k} e^{j}:=d^{3} e^{j}(d x-k) .
$$

We work with $e^{j}$ 's being tensor products of bivariate and univariate mollifiers. With $(v \in \mathbb{N})$

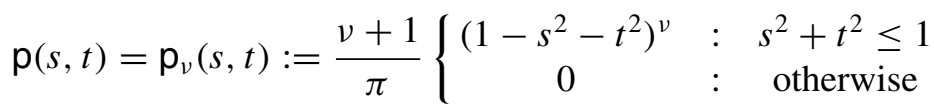

and

$$
\mathrm{q}(s)=\mathrm{q}_{v}(s):=\frac{(2 v+1) ! !}{2^{v+1} v !}\left\{\begin{array}{clc}
\left(1-s^{2}\right)^{v} & : & |s| \leq 1 \\
0 & : & \text { otherwise }^{5}
\end{array}\right.
$$

we define mollifiers adapted to the tensor product structure of the Doppler transform by

$$
\begin{aligned}
e^{1}(x) & :=\mathrm{p}\left(x_{1}, x_{2}\right) \mathrm{q}\left(x_{3}\right), \\
e^{2}(x) & :=\mathrm{p}\left(x_{2}, x_{3}\right) \mathrm{q}\left(x_{1}\right), \\
e^{3}(x) & :=\mathrm{p}\left(x_{1}, x_{3}\right) \mathrm{q}\left(x_{2}\right) .
\end{aligned}
$$

Please note that the mollifiers are normalized, $\int e^{j}(x) \mathrm{d} x=1$, and they are compactly supported but not in $\Omega$. Their supports are cylinders slightly larger than $\Omega$. With the simple rescaling $e^{j}(\cdot):=2^{3 / 2} e^{j}(\sqrt{2} \cdot$ ) (or a rescaling of $p$ and q) we could achieve $e^{j} \in \mathcal{X}_{j}^{\lambda, \lambda}$ for any $\lambda<v+1 / 2$. However, for the reader's convenience we prefer a lean notation and therefore dispense with

$5(2 v+1) ! !=3 \cdot 5 \cdots(2 v+1)$ 
a rescaling. Nevertheless, we will consider the $e^{j}$ 's as elements of $\mathcal{X}_{j}^{\lambda, \lambda}$ for any $\lambda<v+1 / 2$. This minor inaccuracy does not hurt as the mollifiers get scaled anyway, see (3.7).

In Appendix B we prove the mollifier property

$$
\lim _{d \rightarrow \infty}\left\|\mathbf{E}_{d} f-f\right\|_{L^{2}(\Omega)^{3}}=0 \quad \text { for all } f \in L^{2}(\Omega)^{3}
$$

as well as the estimate

$$
\left\|\left(\mathbf{E}_{d}\right)_{j} f-f_{j}\right\|_{L^{2}(\Omega)} \lesssim\left(d^{-\min \{2, \alpha\}}+d^{-\min \{2, \beta\}}\right)\left\|f_{j}\right\|_{\mathcal{X}_{j}^{\alpha, \beta} .}
$$

The kernels $v^{j}$ belonging to the mollifier fields $e^{j} \delta_{j}$ and $\mathbf{D}_{j}, j=1,2,3$ will be explicitely calculated in Section 4 . They satisfy the normal equation

$$
\mathbf{D}_{j} \mathbf{D}_{j}^{*} v^{j}=\mathbf{D}_{j}\left(e^{j} \delta_{j}\right)
$$

which is equivalent to $\mathbf{D}_{j}^{*} v^{j}=P_{\mathrm{N}\left(\mathbf{D}_{j}\right)^{\perp}}\left(e^{j} \delta_{j}\right)$, see (2.9). Finally we have to introduce the reconstruction kernel $v_{d, k}^{j}$ belonging to the mollifier field $e_{d, k}^{j} \delta_{j}$. With the explicit formula for $\mathbf{D}_{j}^{*}$ given in [11, Formula (2.3)] we easily derive the invariance property

$$
\mathbf{D}_{j}^{*} \mathcal{S}_{j}^{d, k}=\mathcal{T}_{j}^{d, k} \mathbf{D}_{j}^{*}
$$

where the translation-dilation operator $\mathcal{S}_{j}^{d, k}$ is defined by

$$
\mathcal{S}_{j}^{d, k} g(\vartheta, s, a):=d^{3} g\left(\vartheta, d s-\left\langle\mathcal{P}_{j}^{*} k, \omega(\vartheta)\right\rangle, d a-\left\langle k, w_{j}\right\rangle\right) .
$$

Define $v_{d, k}^{j}:=\mathcal{S}_{j}^{d, k} v^{j}$. In applying Lemma 2.3 with $A=\mathbf{D}_{j}, S=\mathcal{S}_{j}^{d, k}, T=$ $\mathcal{T}_{j}^{d, k}, X=L_{\Omega}^{2}\left(\mathbb{R}^{3}\right):=\left\{f \in L^{2}\left(\mathbb{R}^{3}\right) \mid \operatorname{supp} f \subset \bar{\Omega}\right\}, Y=L^{2}\left([0,2 \pi] \times \mathbb{R}^{2}\right)$, we obtain

$$
\mathbf{D}_{j}^{*} v_{d, k}^{j}=P_{\mathrm{N}\left(\mathbf{D}_{j}\right)^{\perp}}\left(e_{d, k}^{j} \delta_{j}\right) \quad \text { for } d \geq 1 \text { and } k \in \mathbb{Z}^{3} \text { with }\|k\| \leq d-1 .^{6}
$$

After these preparations we are able to define the approximate inverse $\widetilde{\mathbf{D}}_{j, n_{\ell}, d}^{(\ell)}$ : $\mathbb{R}^{n_{\ell}} \rightarrow L^{2}(\Omega)$ of $\Psi_{p, q, r}^{(\ell)} \mathbf{D}_{j}$, see (2.6) and (2.10):

$$
\widetilde{\mathbf{D}}_{j, n_{\ell}, d}^{(\ell)} w(x):=\sum_{\substack{k \in \mathbb{Z}^{3} \\\|k\| \leq d-1}}\left\langle w, G_{p, q, r}^{(\ell)} \Psi_{p, q, r}^{(\ell)} \mathcal{S}_{j}^{d, k} v^{j}\right\rangle_{\mathbb{R}^{n_{\ell}}} B(d x-k), \quad x \in \Omega,
$$

where $G_{p, q, r}^{(\ell)} \in \mathbb{R}^{n_{\ell} \times n_{\ell}}$ is the Gramian matrix with respect to the spline basis (3.3) $\left(G_{p, q, r}^{(1)}\right.$ is a multiple of the identity matrix). Please note that the inner

${ }^{6}$ The restrictions on $d$ and $k$ guarantee that $\mathcal{T}_{j}^{d, k} \in \mathcal{L}\left(L_{\Omega}^{2}\left(\mathbb{R}^{3}\right)\right)$ which is needed for applying Lemma 2.3. 
products can be evaluated by an algorithm of filtered backprojection type, see [11, Chap. 5] for details and numerical experiments.

Relying on our approximate inverses of the $\Psi_{p, q, r}^{(\ell)} \mathbf{D}_{j}$ 's we find an approximate inverse $\widetilde{\mathbf{D}}_{n_{\ell}, d}^{(\ell)}: \mathbb{R}^{3 n_{\ell}} \rightarrow L^{2}(\Omega)^{3}$ for $\Psi_{p, q, r}^{(\ell)} \mathbf{D}$ by

$$
\left(\widetilde{\mathbf{D}}_{n_{\ell}, d}^{(\ell)} w\right)_{j}(x)=\widetilde{\mathbf{D}}_{j, n_{\ell}, d}^{(\ell)} w_{j}(x) \quad \text { where } w=\left(w_{1}, w_{2}, w_{3}\right)^{t} \text { and } w_{j} \in \mathbb{R}^{n_{\ell}} .
$$

In the remainder of this section we will formulate Theorem 2.2 for $\widetilde{\mathbf{D}}_{j, n_{\ell}, d}^{(\ell)}$ and for $\widetilde{\mathbf{D}}_{n_{\ell}, d}^{(\ell)}$, see Theorem 3.5 and Corollary 3.6 below. Since $\mathbf{D}_{j}$ is not injective we are only able to prove convergence of $\widetilde{\mathbf{D}}_{j, n_{\ell}, d}^{(\ell)} \Psi_{p, q, r}^{(\ell)} \mathbf{D}_{j} f$ to $\left(P_{\left.\mathrm{N}_{\left(\mathbf{D}_{j}\right.}\right)^{\perp}} f\right)_{j}{ }^{7}$

We will need a Sobolev norm estimate for $v_{d, k}^{j}$ which we will establish by a continuity result of $\mathcal{S}_{j}^{d, k}$.

Lemma 3.3 Let $Z=] 0,2 \pi[\times]-1,1\left[, Z_{j}^{d, k}=\left\{\left(\vartheta, d s-\left\langle\mathcal{P}_{j}^{*} k, \omega(\vartheta)\right\rangle\right) \mid\right.\right.$ $(\vartheta, s) \in Z\}$, and $I_{j}^{d, k}=\left\{d a-\left\langle k, w_{j}\right\rangle \mid a \in\right]-1,1[\}$. If $\|k\| \leq d$ then

$$
\left\|\mathcal{S}_{j}^{d, k} g\right\|_{H^{\kappa}(Z) \widehat{\otimes} H^{\lambda}(-1,1)} \lesssim d^{\kappa+\lambda+2}\|g\|_{H^{\kappa}\left(Z_{j}^{d, k}\right) \widehat{\otimes} H^{\lambda}\left(I_{j}^{d, k}\right)}
$$

whenever the right hand side is defined for $\kappa, \lambda \geq 0$.

Proof. We rewrite $\mathcal{S}_{j}^{d, k}$ as a tensor product: $\mathcal{S}_{j}^{d, k}=T_{j}^{d, k} \otimes S_{j}^{d, k}$ where

$$
\begin{aligned}
& T_{j}^{d, k} w(\vartheta, s):=d^{2} w\left(\vartheta, d s-\left\langle\mathcal{P}_{j}^{*} k, \omega(\vartheta)\right\rangle\right) \text { and } \\
& S_{j}^{d, k} u(a):=d u\left(d a-\left\langle k, w_{j}\right\rangle\right) .
\end{aligned}
$$

Now, Lemma 5.3 from [16] applies to $T_{j}^{d, k}$ yielding

$$
\left\|T_{j}^{d, k} w\right\|_{H^{\kappa}(Z)} \lesssim d^{\kappa+3 / 2}\|w\|_{H^{\kappa}\left(Z_{j}^{d, k}\right)} .
$$

Further,

$$
\left\|S_{j}^{d, k} u\right\|_{H^{\lambda}(-1,1)} \lesssim d^{\lambda+1 / 2}\|w\|_{H^{\lambda}\left(I_{j}^{d, k}\right)}
$$

can be validated along the lines of the proof of Lemma 5.3 from [16].

Corollary 3.4 Let the mollifiers $e^{j}, j=1,2,3$, be defined as in (3.10). If $v>\max \{\alpha+1 / 2, \beta-1 / 2\}$ and $d \geq 1$ then

$$
\left\|v_{d, k}^{j}\right\|_{\mathcal{Y}^{\alpha, \beta}} \lesssim d^{\alpha+\beta+5 / 2}
$$

for $j=1,2,3$. The bound is uniform in $k$ with $\|k\| \leq d-1$.

7 The null space of the Doppler transform is explicitely characterized, see [11, Formula (3.15)]. 
Proof. The computation of the reconstruction kernels is outlined in Section 4 below. We will see that every kernel $v^{j}$ corresponding to a mollifier $e^{j}$ from (3.10) has a decomposition like $v^{j}(\vartheta, s, a)=v_{1}^{j}(\vartheta, s, a)+v_{2}^{j}(\vartheta, a)$ with $v_{1}^{j} \in H^{\alpha+1 / 2}((0,2 \pi) \times \mathbb{R}) \widehat{\otimes} H^{\beta}(\mathbb{R})$ and $v_{2}^{j} \in H^{\alpha+1 / 2}(0,2 \pi) \widehat{\otimes} H^{\beta}(\mathbb{R})$, see Lemma 4.1 below. By (3.4), (3.14), and (3.15) we have

$$
\begin{aligned}
\left\|v_{d, k}^{j}\right\|_{\mathcal{Y}^{\alpha, \beta}} \leq & \left\|\mathcal{S}_{j}^{d, k} v_{1}^{j}\right\|_{\mathcal{Y}^{\alpha, \beta}}+\left\|\mathcal{S}_{j}^{d, k} v_{2}^{j}\right\|_{\mathcal{Y}^{\alpha, \beta}} \\
\lesssim & d^{\alpha+\beta+5 / 2}\left\|v_{1}^{j}\right\|_{H^{\alpha+1 / 2}((0,2 \pi) \times \mathbb{R}) \widehat{\otimes} H^{\beta}(\mathbb{R})} \\
& +d^{\beta+5 / 2}\left\|v_{2}^{j}\right\|_{H^{\alpha+1 / 2}(0,2 \pi) \widehat{\otimes} H^{\beta}(\mathbb{R})} \\
\lesssim & d^{\alpha+\beta+5 / 2}
\end{aligned}
$$

which finishes the proof of Corollary 3.4.

Theorem 3.5 Let $\alpha, \beta>1 / 2$ and let $f$ be in $\boldsymbol{X}^{\alpha, \beta}$ with $\operatorname{supp} f \Subset \Omega$. The mollifiers $e^{j}, j=1,2,3$, are defined in (3.10) with $v>\max \{\alpha+1 / 2, \beta-1 / 2\}$. Let $\widetilde{d}=\widetilde{d}(f)$ be the smallest positive integer such that supp $f$ is contained in $\mathcal{B}_{1-1 / \widetilde{d}}(0)$, the ball about the origin with radius $1-1 / \widetilde{d}$.

If $d \geq \widetilde{d}$ then, for $j=1,2,3$,

$$
\begin{aligned}
& \left\|\widetilde{\mathbf{D}}_{j, n_{\ell}, d}^{(\ell)} \Psi_{p, q, r}^{(\ell)} \mathbf{D}_{j} f-\left(P_{\mathrm{N}\left(\mathbf{D}_{j}\right)^{\perp}} f\right)_{j}\right\|_{L^{2}(\Omega)} \\
& \quad \lesssim d^{-\min \{2, \alpha\}}+d^{-\min \{2, \beta\}}+\rho d^{\alpha+\beta+5 / 2} .
\end{aligned}
$$

Proof. For $d \geq \widetilde{d}$ and $\|k\| \geq d$ the inner products $\left\langle f, e_{d, k}^{j} \delta_{j}\right\rangle_{L^{2}(\Omega)^{3}}$ vanish. Thus,

$$
\left(\mathbf{E}_{d}\right)_{j} f(x)=\sum_{\substack{k \in \mathbb{Z}^{3} \\\|k\| \leq d-1}}\left\langle f, e_{d, k}^{j} \delta_{j}\right\rangle_{L^{2}(\Omega)^{3}} B(d x-k) .
$$

Therefore, applying Theorem 2.2 together with Corollary 3.4 we obtain

$$
\begin{aligned}
& \left\|\widetilde{\mathbf{D}}_{j, n_{\ell}, d}^{(\ell)} \Psi_{p, q, r}^{(\ell)} \mathbf{D}_{j} f-\left(P_{\mathrm{N}\left(\mathbf{D}_{j}\right)^{\perp}} f\right)_{j}\right\|_{L^{2}(\Omega)} \\
& \quad \lesssim \rho d^{\alpha+\beta+5 / 2}\|f\|_{X^{\alpha, \beta}}+\left\|\left(\left(I-\mathbf{E}_{d}\right) P_{{\mathrm{N}\left(\mathbf{D}_{j}\right)^{\perp}}} f\right)_{j}\right\|_{L^{2}(\Omega)} .
\end{aligned}
$$

Since $f \in \boldsymbol{X}^{\alpha, \beta}$ we have either $P_{\mathrm{N}\left(\mathbf{D}_{j}\right)} f \in \boldsymbol{X}^{\alpha, \beta}$ or $P_{\mathrm{N}\left(\mathbf{D}_{j}\right)} f \in \boldsymbol{X}^{\alpha, \beta}$ (if both components of $f$ would be less smooth, $f$ would be less smooth). If the former is the case, (3.16) follows immediately from (3.12). In case the latter holds true we proceed with

$$
\left\|\left(\left(I-\mathbf{E}_{d}\right) P_{\mathrm{N}\left(\mathbf{D}_{j}\right)^{\perp}} f\right)_{j}\right\|_{L^{2}(\Omega)}=\left\|\left(\left(I-\mathbf{E}_{d}\right)\left(I-P_{\mathrm{N}\left(\mathbf{D}_{j}\right)^{\perp}}\right) f\right)_{j}\right\|_{L^{2}(\Omega)}
$$

and the triangle inequality together with (3.12) implies (3.16). 
Following we investigate which relations between the data, determined by the parameters $q, p$, and $r$, and the number of reconstruction points, determined by the scaling factor $d$, yield convergence and convergence rates. To formulate the convergence result for $\widetilde{\mathbf{D}}_{n_{\ell}, d}^{(\ell)}$ we introduce the projection $\mathbf{P}: L^{2}(\Omega)^{3} \rightarrow L^{2}(\Omega)^{3}$,

$$
(\mathbf{P} f)_{j}=\left(P_{\mathrm{N}\left(\mathbf{D}_{j}\right)} f\right)_{j}, \quad j=1,2,3 .
$$

Corollary 3.6 Adopt the assumptions of Theorem 3.5. Let $\left\{q_{k}\right\}_{k \in \mathbb{N}},\left\{p_{k}\right\}_{k \in \mathbb{N}}$, $\left\{r_{k}\right\}_{k \in \mathbb{N}}$ be sequences in $\mathbb{N}$ and let $\left\{d_{k}\right\}_{k \in \mathbb{N}}$ be a positive sequence. If all four sequences diverge to infinity and satisfy

$$
\lim _{k \rightarrow \infty} d_{k}^{\alpha+\beta+5 / 2} / \min \left\{q_{k}, p_{k}\right\}^{\min \{\alpha+1 / 2, \ell\}}=\lim _{k \rightarrow \infty} d_{k}^{\alpha+\beta+5 / 2} / r_{k}^{\min \{\beta, \ell\}}=0
$$

then, for $j=1,2,3$,

$$
\lim _{k \rightarrow \infty}\left\|\widetilde{\mathbf{D}}_{j, n_{\ell}, d_{k}}^{(\ell)} \Psi_{p_{k}, q_{k}, r_{k}}^{(\ell)} \mathbf{D}_{j} f-\left(P_{\mathrm{N}\left(\mathbf{D}_{j}\right)^{\perp}} f\right)_{j}\right\|_{L^{2}(\Omega)}=0
$$

as wells as

$$
\lim _{k \rightarrow \infty}\left\|\widetilde{\mathbf{D}}_{n_{\ell}, d_{k}}^{(\ell)} \Psi_{p_{k}, q_{k}, r_{k}}^{(\ell)} \mathbf{D} f-\mathbf{P} f\right\|_{L^{2}(\Omega)^{3}}=0 .
$$

Further, relate $q, p, r$, and $d$ by $p \simeq q, r \simeq q^{\min \{\alpha+1 / 2, \ell\} / \min \{\beta, \ell\}}$, and $d \simeq q^{\lambda{ }^{8}}$ where

$$
\lambda=\lambda(\alpha, \beta)=\frac{\min \{\alpha+1 / 2, \ell\}}{\alpha+\beta+5 / 2+\min \{2, \alpha, \beta\}} .
$$

Then, for $j=1,2,3$,

$$
\left\|\widetilde{\mathbf{D}}_{j, n_{\ell}, d}^{(\ell)} \Psi_{p, q, r}^{(\ell)} \mathbf{D}_{j} f-\left(P_{\mathrm{N}\left(\mathbf{D}_{j}\right)^{\perp}} f\right)_{j}\right\|_{L^{2}(\Omega)} \lesssim q^{-\lambda \min \{2, \alpha, \beta\}}
$$

as well as

$$
\left\|\widetilde{\mathbf{D}}_{n_{\ell}, d}^{(\ell)} \Psi_{p, q, r}^{(\ell)} \mathbf{D} f-\mathbf{P} f\right\|_{L^{2}(\Omega)^{3}} \lesssim q^{-\lambda \min \{2, \alpha, \beta\}}
$$

as $q \rightarrow \infty$.

Proof. First we prove the convergence statements for $\widetilde{\mathbf{D}}_{j, n_{\ell}, d}^{(\ell)}$. The plain convergence follows directly from (3.16) and the assumptions on the sequences $\left\{q_{k}\right\}_{k \in \mathbb{N}},\left\{p_{k}\right\}_{k \in \mathbb{N}},\left\{r_{k}\right\}_{k \in \mathbb{N}}$, and $\left\{d_{k}\right\}_{k \in \mathbb{N}}$.

Now we validate the convergence rate. By assumption we have that $\rho \simeq$ $q^{-\min \{\alpha+1 / 2, \ell\}}$ yielding

$$
\begin{aligned}
& \left\|\widetilde{\mathbf{D}}_{j, n_{\ell}, d}^{(\ell)} \Psi_{p, q, r}^{(\ell)} \mathbf{D}_{j} f-\left(P_{\mathrm{N}\left(\mathbf{D}_{j}\right)^{\perp}} f\right)_{j}\right\|_{L^{2}(\Omega)} \\
& \quad \lesssim q^{-\lambda \min \{2, \alpha\}}+q^{-\lambda \min \{2, \beta\}}+q^{-\min \{\alpha+1 / 2, \ell\}+\lambda(\alpha+\beta+5 / 2)} .
\end{aligned}
$$

\footnotetext{
${ }^{8}$ By $A \simeq B$ we abbreviate the two-sided inequality $A \lesssim B \lesssim A$.
} 
Since $-\min \{\alpha+1 / 2, \ell\}+\lambda(\alpha+\beta+5 / 2)=-\lambda \min \{2, \alpha, \beta\}$ the convergence rate is established.

The corresponding results for $\widetilde{\mathbf{D}}_{n_{\ell}, d}^{(\ell)}$ are readily obtained by the first parts of the proof using that the norm on $L^{2}(\Omega)^{3}$ is given by $\|f\|_{L^{2}(\Omega)^{3}}^{2}=$ $\sum_{j=1}^{3}\left\|f_{j}\right\|_{L^{2}(\Omega)}^{2}$.

We end this section by a discussion of the regularization power of $\widetilde{\mathbf{D}}_{j, n_{\ell}, d}^{(\ell)}$. Corollary 3.7 below is based on the abstract result from [16, Section 4.2]. By $\Psi_{p, q, r}^{(\ell), \delta}: \mathcal{Y}^{\alpha, \beta} \rightarrow \mathbb{R}^{n_{\ell}}$ we denote a perturbed (noisy) realization of the observation operator with a relative noise level $\delta>0$ :

$$
\left|\left(\Psi_{p, q, r}^{(\ell), \delta} y\right)_{i, j, k}-\left(\Psi_{p, q, r}^{(\ell)} y\right)_{i, j, k}\right| \leq \delta\|y\|_{\mathcal{Y}^{\alpha, \beta}} .
$$

Corollary 3.7 Adopt the assumptions of Theorem 3.5. Suppose that $p \simeq q$, $r \simeq q^{\min \{\alpha+1 / 2, \ell\} / \min \{\beta, \ell\}}$, and $d \simeq q^{\lambda}$ with $\lambda$ from (3.17). Further, assume (3.18) holds true. If $q \simeq \delta^{-1 / \min \{\alpha+1 / 2, \ell\}}$ then, for $j=1,2,3$,

$$
\left\|\widetilde{\mathbf{D}}_{j, n_{\ell}, d}^{(\ell)} \Psi_{p, q, r}^{(\ell), \delta} \mathbf{D}_{j} f-\left(P_{\mathrm{N}\left(\mathbf{D}_{j}\right)^{\perp}} f\right)_{j}\right\|_{L^{2}(\Omega)} \lesssim \delta^{\frac{\min \{2, \alpha, \beta\}}{\alpha+\beta+5 / 2+\min \{2, \alpha, \beta\}}}
$$

as well as

$$
\left\|\widetilde{\mathbf{D}}_{n_{\ell}, d}^{(\ell)} \Psi_{p, q, r}^{(\ell), \delta} \mathbf{D} f-\mathbf{P} f\right\|_{L^{2}(\Omega)^{3}} \lesssim \delta^{\frac{\min \{2, \alpha, \beta\}}{\alpha+\beta+5 / 2+\min \{2, \alpha, \beta\}}}
$$

as $\delta \rightarrow 0$.

Proof. We follow a standard procedure from regularization theory and split the reconstruction error in the approximation error and the data error:

$$
\begin{aligned}
& \left\|\widetilde{\mathbf{D}}_{j, n_{\ell}, d}^{(\ell)} \Psi_{p, q, r}^{(\ell), \delta} \mathbf{D}_{j} f-\left(P_{\mathrm{N}\left(\mathbf{D}_{j}\right)^{\perp}} f\right)_{j}\right\|_{L^{2}(\Omega)} \\
& \leq \| \widetilde{\mathbf{D}}_{j, n_{\ell}}^{(\ell)} \Psi_{p, q, r}^{(\ell)} \mathbf{D}_{j} f-\left(P_{\left.\mathrm{N}\left(\mathbf{D}_{j}\right)^{\perp} f\right)_{j} \|_{L^{2}(\Omega)}}\right. \\
& \quad+\left\|\widetilde{\mathbf{D}}_{j, n_{\ell}, d}^{(\ell)}\left(\Psi_{p, q, r}^{(\ell), \delta}-\Psi_{p, q, r}^{(\ell)}\right) \mathbf{D}_{j} f\right\|_{L^{2}(\Omega)} .
\end{aligned}
$$

According to the proof of Theorem 4.3 from [16] the data error is bounded by a multiple of $\delta d^{\alpha+\beta+5 / 2}=\delta q^{\lambda(\alpha+\beta+5 / 2)}$. The approximation error has already be bounded in Corollary 3.6 by a multiple of $q^{-\lambda \min \{2, \alpha, \beta\}}$, that is,

$$
\left\|\widetilde{\mathbf{D}}_{j, n_{\ell}, d}^{(\ell)} \Psi_{p, q, r}^{(\ell), \delta} \mathbf{D}_{j} f-\left(P_{\mathrm{N}\left(\mathbf{D}_{j}\right)^{\perp}} f\right)_{j}\right\|_{L^{2}(\Omega)} \lesssim q^{-\lambda \min \{2, \alpha, \beta\}}+\delta q^{\lambda(\alpha+\beta+5 / 2)}
$$

Balancing the terms on the right verifies Corollary 3.7. 


\section{Computing the reconstruction kernel}

We analytically solve the normal equation (3.13) for $e^{j}$ defined in (3.10). Schuster [10, Corollary 3.4] discovered that the solution $v^{j}$ of (3.13) can be determined by

$$
\left(\mathbf{R}^{*} \otimes I\right) \partial_{s} v^{j}=w_{j} \cdot \nabla \times\left(e^{j} \delta_{j}\right)
$$

where $\mathbf{R}^{*}$ is the adjoint operator of the $2 \mathrm{D}$-Radon transform $\mathbf{R}: L^{2}\left(\Omega_{2}\right) \rightarrow$ $L^{2}(Z)$ given by $\left(\Omega_{2}\right.$ is the unit disk in $\left.\mathbb{R}^{2}\right)$

$$
\mathbf{R} f(\vartheta, s):=\int_{L(\vartheta, s) \cap \Omega_{2}} f(x) \mathrm{d} \sigma(x)
$$

mapping a function to its integrals over the lines $L(\vartheta, s)=\left\{\tau \omega^{\perp}(\vartheta)+\right.$ $s \omega(\vartheta) \mid \tau \in \mathbb{R}\}$ where $s \in \mathbb{R}, \omega(\vartheta)=(\cos \vartheta, \sin \vartheta)^{t}$, and $\omega^{\perp}(\vartheta)=$ $(-\sin \vartheta, \cos \vartheta)^{t}$ for $\left.\vartheta \in\right] 0,2 \pi[$.

Since the three reconstruction kernels $v^{j}, j=1,2,3$ are easily related we restrict our attention to the case $j=1$ in which (4.1) reduces to

$$
\left(\mathbf{R}^{*} \otimes I\right) \partial_{s} v^{1}=-\partial_{x_{2}} e^{1} .
$$

We extend the ideas from [14] for calculating reconstruction kernels for the 2D-Radon transform to the Doppler transform. Starting point is the well known inversion formula

$$
f=(4 \pi)^{-1} \mathbf{R}^{*} \Lambda \mathbf{R} f
$$

for $f \in L^{2}\left(\Omega_{2}\right)$, see, e.g., Natterer [12]. Here, $\Lambda$ denotes the Riesz potential: $\widehat{\Lambda u}(\sigma)=|\sigma| \widehat{u}(\sigma)$ and acts only on the second variable of $\mathbf{R} f$. By (4.3) we can solve (4.2) for $\partial_{s} v^{1}$. Indeed,

$$
\partial_{s} v^{1}=-(4 \pi)^{-1}(\Lambda \mathbf{R} \otimes I) \partial_{x_{2}} e^{1}=-(4 \pi)^{-1}\left(\Lambda \mathbf{R} \partial_{x_{2}} p\right) \otimes q .
$$

For evaluating $\Lambda \mathbf{R} \partial_{x_{2}} p$ we rely on the projection slice theorem [12, Chap. II.1, Th. 1.1]:

$$
\widehat{\mathbf{R} f}(\vartheta, \sigma)=\sqrt{2 \pi} \widehat{f}(\sigma \omega(\vartheta)) \cdot{ }^{9}
$$

By the inverse 1D-Fourier transform and the radial symmetry of $p$ we find that

${ }^{9}$ On the left we have a 1D-Fourier transform and on the right we have a 2D-Fourier transform. 


$$
\begin{aligned}
\Lambda \mathbf{R} \partial_{x_{2}} \mathrm{p}(\vartheta, s) & =\int_{\mathbb{R}}|\sigma| \widehat{\partial_{x_{2}} \mathrm{p}}(\sigma \omega(\vartheta)) \mathrm{e}^{\imath s \sigma} \mathrm{d} \sigma \\
& =\imath \sin \vartheta \int_{\mathbb{R}}|\sigma| \sigma \widehat{\mathrm{p}}(\sigma \omega(0)) \mathrm{e}^{\imath s \sigma} \mathrm{d} \sigma \\
& =-2 \sin \vartheta \int_{0}^{\infty} \sigma^{2} \widehat{\mathrm{p}}(\sigma, 0) \sin (s \sigma) \mathrm{d} \sigma
\end{aligned}
$$

Thus,

$$
\partial_{s} v^{1}(\vartheta, s, a)=\frac{1}{2 \pi} \sin \vartheta \mathbf{q}(a) \int_{0}^{\infty} \sigma^{2} \widehat{\mathbf{p}}(\sigma, 0) \sin (s \sigma) \mathrm{d} \sigma .
$$

From [14, Example 3.2] we know that

$$
\widehat{\mathbf{p}}(\sigma, 0)=\frac{(v+1) !}{2 \pi}\left(\frac{2}{\sigma}\right)^{\nu+1} \mathbf{J}_{v+1}(\sigma)
$$

where $\mathrm{J}_{n}$ is the Bessel function of the first kind of order $n$. Finally, we obtain

$$
\begin{aligned}
& \partial_{s} v^{1}(\vartheta, s, a) \\
& =\frac{1}{\pi^{2}}(v+1) ! 2^{\nu-1} \sin \vartheta \mathbf{q}(a) \int_{0}^{\infty} \sigma^{1-v} \mathbf{J}_{v+1}(\sigma) \sin (s \sigma) \mathrm{d} \sigma \\
& =\frac{1}{\pi^{2}} \sin \vartheta \mathrm{q}(a)\left\{\begin{array}{cc}
2 v(v+1) s \mathrm{~F}\left(2,1-v ; \frac{3}{2} ; s^{2}\right) & :|s|<1 \\
-\frac{1}{2} s^{-3} \mathrm{~F}\left(2, \frac{3}{2} ; v+2 ; s^{-2}\right) & :|s| \geq 1
\end{array}\right.
\end{aligned}
$$

where we used Formula (6.699.1) from [2] for evaluating the integral. Here, $\mathrm{F}={ }_{2} \mathrm{~F}_{1}$ denotes the hypergeometric series. Hence, the searched-for reconstruction kernel $v^{1}$ has a representation

$$
v^{1}(\vartheta, s, a)=\mathrm{I}(\vartheta, s, a)+h(\vartheta, a) .
$$

where

$$
\mathrm{I}(\vartheta, s, a):=\int_{-1}^{s} \partial_{s} v^{1}(\vartheta, t, a) \mathrm{d} t
$$

and $h$ is an integration constant which does not depend on $s$. We are now heading for explicit expressions for I and $h$.

We first look at $h$. The proof of Theorem 3.9 in [10] gives that

$$
\mathcal{M}:=\{\sin k \vartheta, \cos k \vartheta \mid k \in \mathbb{N} \cup\{0\}, k \neq 1\} \perp \overline{\mathrm{R}\left(\mathbf{D}_{j}\right)} .
$$

Since $v^{1}$ is the unique solution of the normal equation (3.13) in $\overline{\mathrm{R}\left(\mathbf{D}_{1}\right)}$, the orthogonality (4.9) implies

$$
\int_{0}^{2 \pi} v^{1}(\vartheta, s, a) \sin (k \vartheta) \mathrm{d} \vartheta=\int_{0}^{2 \pi} v^{1}(\vartheta, s, a) \cos (k \vartheta) \mathrm{d} \vartheta=0
$$

for $k \in \mathbb{N} \cup\{0\}, k \neq 1$. Moreover, in view of (4.6), 


$$
\int_{0}^{2 \pi} \mathrm{I}(\vartheta, s, a) \sin (k \vartheta) \mathrm{d} \vartheta=\int_{0}^{2 \pi} \mathrm{I}(\vartheta, s, a) \cos (k \vartheta) \mathrm{d} \vartheta=0
$$

for $k \in \mathbb{N} \cup\{0\}, k \neq 1$. Both latter displayed formulas together with (4.7) lead to

$$
\int_{0}^{2 \pi} h(\vartheta, a) \sin (k \vartheta) \mathrm{d} \vartheta=\int_{0}^{2 \pi} h(\vartheta, a) \cos (k \vartheta) \mathrm{d} \vartheta=0
$$

for $k \in \mathbb{N} \cup\{0\}, k \neq 1$. Further, we claim that

$$
\int_{0}^{2 \pi} h(\vartheta, a) \cos \vartheta \mathrm{d} \vartheta=0 .
$$

Once we have computed the Fourier coefficient $\int_{0}^{2 \pi} h(\varphi, a) \sin \varphi \mathrm{d} \varphi$ the function $h$ obviously reads

$$
h(\vartheta, a)=\frac{1}{\pi} \int_{0}^{2 \pi} h(\varphi, a) \sin \varphi \mathrm{d} \varphi \sin (\vartheta)
$$

because $\mathcal{M} \cup\{\cos \vartheta, \sin \vartheta\}$ forms a complete orthogonal system in $L^{2}(0,2 \pi)$.

To verify (4.10) we rely on the normal equation (3.13) setting $j=1$, $\vartheta=0$, and $s=0$ :

$$
\mathbf{D}_{1} \mathbf{D}_{1}^{*} h(0,0, a)=\mathbf{D}_{1}\left(e^{1} \delta_{1}\right)(0,0, a)-\mathbf{D}_{1} \mathbf{D}_{1}^{*} \mathrm{I}(0,0, a) .
$$

It is easily checked that $\mathbf{D}_{1}\left(e^{1} \delta_{1}\right)(0,0, a)=0$. Therefore, (4.11) reduces to

$$
2 \int_{0}^{2 \pi} h(\vartheta, a) \cos \vartheta \mathrm{d} \vartheta=-\int_{0}^{2 \pi} \int_{-1}^{1} \mathrm{I}(\vartheta, t \sin \vartheta, a) \cos \vartheta \mathrm{d} t \mathrm{~d} \vartheta
$$

Observing that $\mathrm{I}(-\vartheta,-t \sin \vartheta, a)=-\mathrm{I}(\vartheta, t \sin \vartheta, a)$ the latter integral vanishes. Thus, (4.10) is established.

To calculate the Fourier coefficient of $h$ with respect to $\sin (\vartheta)$ we set $j=1, \vartheta=\pi / 2$ and $s=0$ in (3.13). A little bit of analysis shows that

$$
\mathbf{D}_{1}\left(e^{1} \delta_{1}\right)(\pi / 2,0, a)=-\mathrm{q}(a) \int_{-1}^{1}\left(1-t^{2}\right)^{v} \mathrm{~d} t=-\frac{2^{v+1} ! v}{(2 v+1) ! !} \mathrm{q}(a)
$$

and

$$
\mathbf{D}_{1} \mathbf{D}_{1}^{*} \mathrm{I}(\pi / 2,0, a)=\int_{0}^{2 \pi} \int_{-1}^{1} \mathrm{I}(\vartheta, t \cos \vartheta, a) \sin \vartheta \mathrm{d} t \mathrm{~d} \vartheta .
$$

Further,

$$
\mathbf{D}_{1} \mathbf{D}_{1}^{*} h(\pi / 2,0, a)=2 \int_{0}^{2 \pi} h(\vartheta, a) \sin \vartheta \mathrm{d} \vartheta
$$


and we finally found the following representation of the integration constant $h$ :

$$
\begin{aligned}
h(\vartheta, a)= & -\frac{1}{2 \pi} \sin \vartheta\left(\frac{2^{v+1} v !}{(2 v+1) ! !} \mathrm{q}(a)\right. \\
& \left.+\int_{0}^{2 \pi} \int_{-1}^{1} \mathrm{I}(\vartheta, t \cos \vartheta, a) \mathrm{d} t \sin \vartheta \mathrm{d} \vartheta\right) .
\end{aligned}
$$

To calculate the integrals in (4.13) we first determine I explicitly. We distinguish the three cases $|s|<1, s \geq 1$, and $s \leq-1$.

In case of $|s|<1$ we have

$$
\begin{aligned}
\mathrm{I}(\vartheta, s, a) & =\int_{-1}^{s} \partial_{s} v^{1}(\vartheta, t, a) \mathrm{d} t \\
& =\frac{2}{\pi^{2}} v(v+1) \sin \vartheta \mathrm{q}(a) \int_{-1}^{s} t \mathrm{~F}\left(2,1-v ; \frac{3}{2} ; t^{2}\right) \mathrm{d} t .
\end{aligned}
$$

Using the series representation

$$
\mathrm{F}(\alpha, \beta ; \delta ; z)=\sum_{k=0}^{\infty} \frac{(\alpha)_{k}(\beta)_{k}}{(\delta)_{k} k !} z^{k}
$$

with the Pochhammer symbols $(\alpha)_{k}=\alpha \cdot(\alpha+1) \cdot \ldots \cdot(\alpha+k-1)$ if $k>0$ and $(\alpha)_{k}=1$, otherwise, we get from (4.14)

$$
\begin{aligned}
\mathrm{I}(\vartheta, s, a)= & \frac{1}{\pi^{2}} v(v+1) \sin \vartheta \mathrm{q}(a) \\
& \times\left(s^{2} \mathrm{~F}\left(1,1-v ; \frac{3}{2} ; s^{2}\right)-\mathrm{F}\left(1,1-v ; \frac{3}{2} ; 1\right)\right) \text { for }|s|<1 .
\end{aligned}
$$

Note that in this case $\mathrm{I}$ is a polynomial in $s$ since $1-v \leq 0$. A similar calculation for $s \geq 1$ leads to

$$
\begin{aligned}
\mathrm{I}(\vartheta, s, a)= & -\frac{1}{4 \pi^{2}} \sin \vartheta \mathrm{q}(a) \\
& \times\left(\mathrm{F}\left(1, \frac{3}{2} ; v+2 ; 1\right)-s^{-2} \mathrm{~F}\left(1, \frac{3}{2} ; v+2 ; s^{-2}\right)\right) .
\end{aligned}
$$

In case of $s \leq-1$ the symmetry

$$
\int_{-1}^{s} \partial_{s} v^{1}(\vartheta, t, a) \mathrm{d} t=\int_{1}^{-s} \partial_{s} v^{1}(\vartheta, t, a) \mathrm{d} t
$$

yields the same representation for I as in (4.15). With $\mathrm{F}\left(1,1-v ; \frac{3}{2} ; 1\right)=$ $1 /(2 v-1)$ and $\mathrm{F}\left(1, \frac{3}{2} ; v+2 ; 1\right)=(2 v+2) /(2 v-1)$ the integral $\mathrm{I}$ is explicitely represented by 
$\mathrm{I}(\vartheta, s, a)=\frac{1}{\pi^{2}} \sin \vartheta \mathrm{q}(a)$

$$
\times\left\{\begin{array}{c}
v(v+1)\left(s^{2} \mathrm{~F}\left(1,1-v ; \frac{3}{2} ; s^{2}\right)-1 /(2 v-1)\right):|s|<1 \\
\frac{1}{4}\left(s^{-2} \mathrm{~F}\left(1, \frac{3}{2} ; v+2 ; s^{-2}\right)-(2 v+2) /(2 v-1)\right):|s| \geq 1
\end{array} .\right.
$$

Since

$$
\begin{aligned}
\int_{0}^{z} t^{2} \mathrm{~F}\left(1,1-v ; \frac{3}{2} ; t^{2}\right) \mathrm{d} t & =\sum_{k=0}^{v-1} \frac{(1-v)_{k}}{(3 / 2)_{k}} \int_{0}^{z} t^{2 k+2} \mathrm{~d} t \\
& =\sum_{k=0}^{v-1} \frac{(1-v)_{k}}{(3 / 2)_{k}(2 k+3)} z^{2 k+3}
\end{aligned}
$$

we derive from (4.16)

$$
\begin{aligned}
\int_{-1}^{1} \mathrm{I}(\vartheta, t \cos \vartheta, a) \mathrm{d} t= & \frac{2}{\pi^{2}} v(v+1) \sin \vartheta q(a)\left(\sum_{k=0}^{v-1} \frac{(1-v)_{k}}{(3 / 2)_{k}(2 k+3)}\right. \\
& \left.\times(\cos \vartheta)^{2 k+2}-(2 v-1)^{-1}\right) .
\end{aligned}
$$

With an integration by parts and Formula (3.631.17) from [2] we get

$$
\int_{0}^{2 \pi} \sin ^{2} \vartheta(\cos \vartheta)^{2 k+2} \mathrm{~d} \vartheta=\frac{\pi}{(2 k+3) 2^{2 k+3}}\left(\begin{array}{c}
2 k+4 \\
k+2
\end{array}\right)
$$

which finally gives

$$
\begin{aligned}
& \int_{0}^{2 \pi} \int_{-1}^{1} \mathrm{I}(\vartheta, t \cos \vartheta, a) \mathrm{d} t \sin \vartheta \mathrm{d} \vartheta \\
& \quad=\frac{2}{\pi} v(v+1) \mathrm{q}(a)\left(\sum_{k=0}^{v-1} \frac{(1-v)_{k}\left(\begin{array}{c}
2 k+4 \\
k+2
\end{array}\right)}{(3 / 2)_{k}(2 k+3)^{2} 2^{2 k+3}}-\frac{1}{2 v-1}\right) .
\end{aligned}
$$

By (4.7), (4.13), (4.16), and (4.17) we find the following representation of the solution $v^{1}$ of $(3.13)$ in $\overline{\mathrm{R}\left(\mathbf{D}_{1}\right)}$ :

$$
\begin{aligned}
v^{1}(\vartheta, s, a)= & \frac{1}{\pi^{2}} \sin \vartheta \mathrm{q}(a) \\
& \times\left\{\begin{array}{c}
v(v+1)\left(s^{2} \mathrm{~F}\left(1,1-v ; \frac{3}{2} ; s^{2}\right)-\frac{1}{2 v-1}\right)-c_{v}:|s|<1 \\
\frac{1}{4}\left(s^{-2} \mathrm{~F}\left(1, \frac{3}{2} ; v+2 ; s^{-2}\right)-\frac{2 v+2}{2 v-1}\right)-c_{v} \quad:|s| \geq 1
\end{array}\right.
\end{aligned}
$$

where

$$
c_{v}=v(v+1)\left(\sum_{k=0}^{v-1} \frac{(1-v)_{k}\left(\begin{array}{c}
2 k+4 \\
k+2
\end{array}\right)}{(3 / 2)_{k}(2 k+3)^{2} 2^{2 k+3}}-\frac{1}{2 v-1}\right)+\pi \frac{2^{v} v !}{(2 v+1) ! !} .
$$

Analogously we obtain $v^{2}=v^{1}$. The third kernel $v^{3}$ emerges from $v^{1}$ by replacing $\sin \vartheta$ by $\cos \vartheta$. See Figures 1 and 2 for graphical displays of $v^{1}$.

In our convergence analysis of the approximate inverse for the Doppler transform in Sect. 3.2 we used the following kernel splitting. 


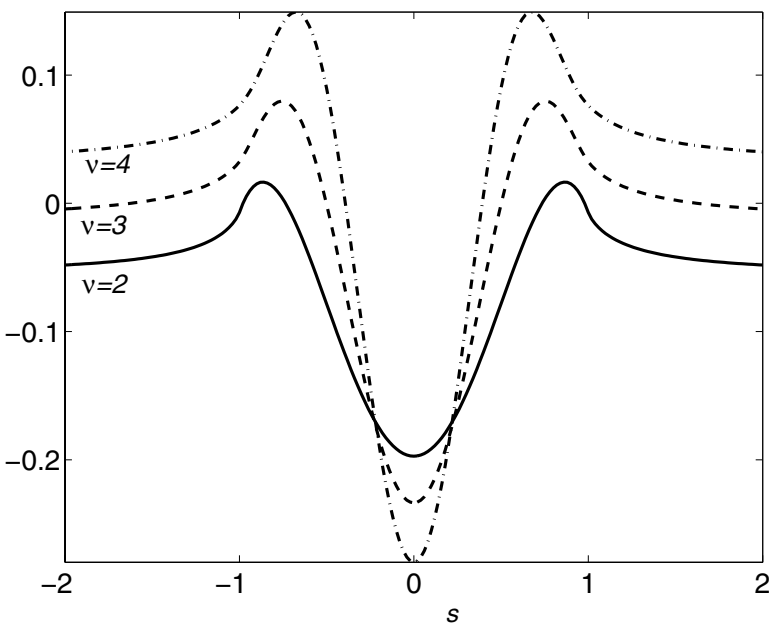

Fig. 1. 1D-Cross sections of the reconstruction kernel (4.18): $v^{1}(\pi / 2, \cdot, 0)$ for $v=2$ (solid line), $v=3$ (dashed line), and $v=4$ (dashed-dotted line)

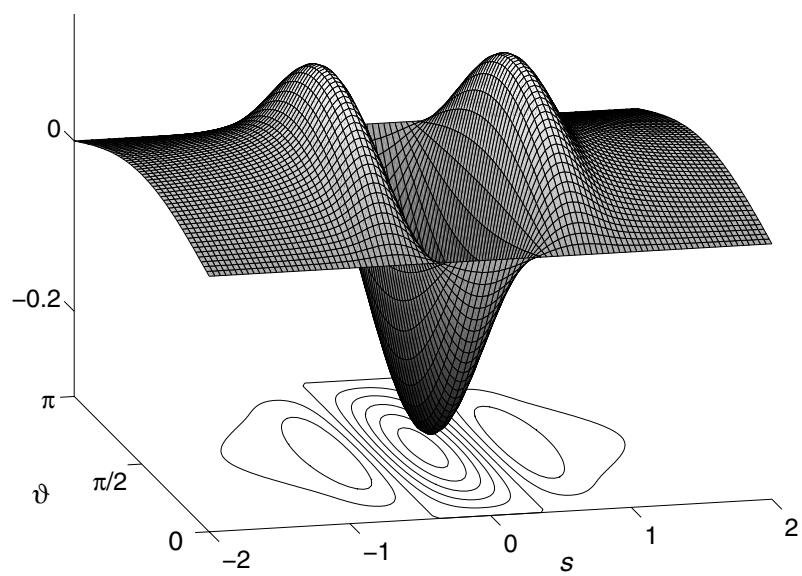

Fig. 2. 2D-Cross section of the reconstruction $\operatorname{kernel}(4.18): v^{1}(\cdot, \cdot, 0)$ for $v=4$

Lemma 4.1 Let $v^{j}$ be the reconstruction kernel belonging to $e^{j}$ as in (3.10). Then, $v^{j}$ can be split according to

$$
v^{j}(\vartheta, s, a)=v_{1}^{j}(\vartheta, s, a)+v_{2}^{j}(\vartheta, a)
$$

where

$$
v_{1} \in H^{r}((0,2 \pi) \times \mathbb{R}) \widehat{\otimes} H_{0}^{t}(-1,1), \quad r<v, t<v+1 / 2,
$$

and 


$$
v_{2} \in H^{r}(0,2 \pi) \widehat{\otimes} H_{0}^{t}(-1,1), \quad r \geq 0, t<v+1 / 2 .
$$

Proof. Without loss of generality we restrict ourselves to $j=1$. The stated splitting follows from rearranging (4.7). To see this we write

$$
\mathrm{I}(\vartheta, s, a)=\kappa \sin \vartheta \mathrm{q}(a) W(s)
$$

where $\kappa$ is a constant and

$$
W(s):=\int_{-1}^{s} w(t) \mathrm{d} t \quad \text { with } w(t)=\int_{0}^{\infty} \sigma^{1-v} \mathbf{J}_{v+1}(\sigma) \sin (t \sigma) \mathrm{d} \sigma,
$$

see (4.8) and (4.6). Further, by (4.4) and (4.5),

$$
w(s) \sin \vartheta=\rho \Lambda \mathbf{R} \partial_{x_{2}} \mathrm{p}(\vartheta, s)
$$

for a suitable constant $\rho$. Both, $\mathbf{R}: H_{0}^{r}\left(\Omega_{2}\right) \rightarrow L^{2}(0,2 \pi) \widehat{\otimes} H^{r+1 / 2}(\mathbb{R})$ and $\Lambda: H^{r}(\mathbb{R}) \rightarrow H^{r-1}(\mathbb{R})$ are bounded operators for any $r \geq 0$, see Louis and Natterer [9, Theorem 3.1]. Since $\mathrm{p}$ is in $H_{0}^{\beta}\left(\Omega_{2}\right)$ for any $\beta<v+1 / 2$ we have that

$$
w \in H^{r}(\mathbb{R}) \quad \text { for any } r<v-1 .
$$

Moreover,

$$
\widetilde{W}(\cdot):=W(\cdot)+\frac{1}{4} \frac{2 v+2}{2 v-1} \text { is in } L^{2}(\mathbb{R})
$$

as inspecting (4.16) shows. Now, $\partial_{s} \widetilde{W}=w \in H^{r}(\mathbb{R}), r<v-1$, together with $\widetilde{W} \in L^{2}(\mathbb{R})$ yields $\widetilde{W} \in H^{r}(\mathbb{R})$ for any $r<v$. Setting

$$
v_{1}^{1}=\kappa \sin \vartheta \mathrm{q}(a) \widetilde{W}(s) \quad \text { and } \quad v_{2}^{1}=h(\vartheta, a)-\frac{\kappa}{4} \frac{2 v+2}{2 v-1} \sin \vartheta \mathrm{q}(a)
$$

and recalling that $h(\vartheta, a)=c \sin \vartheta \mathrm{q}(a)$ with a constant $c$ we finally found the kernel splitting with the stated smoothness properties.

\section{A Appendix: proof of uniform boundedness (3.4) and approximation property (3.5)}

Observe that $\Pi_{p, q, r}^{(\ell)}=\Pi_{p, q}^{(\ell)} \otimes \Pi_{r}^{(\ell)}$ where $\Pi_{p, q}^{(\ell)}: H^{\kappa+1}(Z) \rightarrow S_{\vartheta}^{(\ell)} \otimes S_{s}^{(\ell)}$ and $\Pi_{r}^{(\ell)}: H^{\kappa+1 / 2}(-1,1) \rightarrow S_{a}^{(\ell)}$ with $\kappa>0$ are defined by

$$
\Pi_{p, q}^{(\ell)} y:=\sum_{j=0}^{p_{\ell}} \sum_{i=-q}^{q_{\ell}}\left(\Psi_{p, q}^{(\ell)} y\right)_{j, i} B_{p, j}^{(\ell)} \otimes B_{q, i}^{(\ell)} \quad \text { and } \quad \Pi_{r}^{(\ell)} y:=\sum_{k=-r}^{r_{\ell}}\left(\Psi_{r}^{(\ell)} y\right)_{k} B_{r, k}^{(\ell)},
$$

see Sect. 3.2 for the notation. For $\alpha>1$ and $\beta>1 / 2$ we have

$$
\text { (A.1) }\left\|\Pi_{q, p}^{(\ell)}\right\|_{H^{\alpha}(Z) \rightarrow L^{2}(Z)} \lesssim 1 \text { and }\left\|\Pi_{r}^{(\ell)}\right\|_{H^{\beta}(-1,1) \rightarrow L^{2}(-1,1)} \lesssim 1
$$

as well as 
(A.2a)

$$
\left\|I-\Pi_{q, p}^{(\ell)}\right\|_{H^{\alpha}(Z) \rightarrow L^{2}(Z)} \lesssim \max \left\{h_{\vartheta}, h_{s}\right\}^{\min \{\alpha, \ell\}}
$$

and

$$
\left\|I-\Pi_{r}^{(\ell)}\right\|_{H^{\beta}(-1,1) \rightarrow L^{2}(-1,1)} \lesssim h_{a}^{\min \{\beta, \ell\}}
$$

whenever the right hand sides are finite. The constants involved do not depend on $p, q$, and $r$. All four estimates in (A.1) and (A.2) are standard results from spline approximation theory, see, e.g., Schumaker [17, Chap. 12].

The norm of a tensor product operator is dominated by the product of the norms of its factors, see, e.g., Aubin [1, Prop. 12.4.1]. Hence,

$$
\left\|\Pi_{p, q, r}^{(\ell)}\right\|_{\mathcal{Y} \alpha, \beta \rightarrow L^{2}(Q)} \leq\left\|\Pi_{p, q}^{(\ell)}\right\|_{H^{\alpha+1 / 2}(Z) \rightarrow L^{2}(Z)}\left\|\Pi_{r}^{(\ell)}\right\|_{H^{\beta}(-1,1) \rightarrow L^{2}(-1,1)}
$$

and the boundedness (3.4) follows immediately from (A.1). The approximation property (3.5) is implied by

$$
\begin{aligned}
& \left\|\Pi_{p, q, r}^{(\ell)}-I\right\|_{\mathcal{Y}^{\alpha, \beta} \rightarrow L^{2}(Q)} \\
& \quad=\left\|\Pi_{p, q, r}^{(\ell)}-I \otimes I\right\|_{\mathcal{Y}^{\alpha, \beta} \rightarrow L^{2}(Q)} \\
& \quad \leq\left\|\Pi_{p, q}^{(\ell)} \otimes\left(\Pi_{r}^{(\ell)}-I\right)\right\|_{\mathcal{Y}^{\alpha, \beta} \rightarrow L^{2}(Q)}+\left\|\left(\Pi_{p, q}^{(\ell)}-I\right) \otimes I\right\|_{\mathcal{Y}^{\alpha, \beta} \rightarrow L^{2}(Q)} \\
& \quad \lesssim\left\|I-\Pi_{r}^{(\ell)}\right\|_{H^{\beta}(-1,1) \rightarrow L^{2}(-1,1)}+\left\|I-\Pi_{q, p}^{(\ell)}\right\|_{H^{\alpha+1 / 2}(Z) \rightarrow L^{2}(Z)}
\end{aligned}
$$

when applying (A.2).

\section{B Appendix: mollifier property - proof of (3.11) and (3.12)}

Here we will also exploit the tensor product structure of $\left(\mathbf{E}_{d}\right)_{j}$. Therefore, we introduce the auxiliary operators $E_{d}^{(i)}: L^{2}\left(\mathbb{R}^{i}\right) \rightarrow L^{2}\left(\mathbb{R}^{i}\right), i=1,2, d>0$, by

$$
E_{d}^{(2)} u:=\sum_{k \in \mathbb{Z}^{2}}\left\langle u, \mathrm{p}_{d, k}\right\rangle_{L^{2}\left(\mathbb{R}^{2}\right)} b \otimes b(d \cdot-k)
$$

and

$$
E_{d}^{(1)} w:=\sum_{l \in \mathbb{Z}}\left\langle w, \mathrm{q}_{d, l}\right\rangle_{L^{2}(\mathbb{R})} b(d \cdot-l)
$$

where $b$ is the univariate linear B-spline supported in $[-1,1]$. Further,

$$
\mathrm{p}_{d, k}(s, t)=d^{2} \mathrm{p}(d s-k) \quad \text { and } \quad \mathrm{q}_{d, l}(s)=d \mathrm{q}(d s-l) .
$$

The functions $p$ and $q$ are those from (3.8) and (3.9), respectively. In [16, Appendix B] we proved the convergence estimates

$$
\left\|E_{d}^{(i)}-I\right\|_{H^{\alpha}\left(\mathbb{R}^{i}\right) \rightarrow L^{2}\left(\mathbb{R}^{i}\right)} \lesssim d^{-\min \{2, \alpha\}}, \quad \alpha \geq 0, \quad i=1,2 .
$$


Note that

$$
\left(\mathbf{E}_{d}\right)_{j} f=E_{d}^{(2)} \otimes E_{d}^{(1)}\left(f_{j} \circ \mathrm{Q}_{j}\right), \quad j=1,2,3,
$$

with the permutations $\mathrm{Q}_{j}\left(x_{1}, x_{2}, x_{3}\right):=\mathcal{P}_{j}\left(x_{1}, x_{2}\right)+x_{3} w_{j}$. Arguing as in the end of Appendix A we find by (B.1) that

$$
\begin{aligned}
& \left\|E_{d}^{(2)} \otimes E_{d}^{(1)}-I\right\|_{H^{\alpha}\left(\mathbb{R}^{2}\right) \widehat{\otimes} H^{\beta}(\mathbb{R}) \rightarrow L^{2}\left(\mathbb{R}^{3}\right)} \\
& \quad \lesssim\left\|E_{d}^{(2)}-I\right\|_{H^{\alpha}\left(\mathbb{R}^{2}\right) \rightarrow L^{2}\left(\mathbb{R}^{2}\right)}+\left\|E_{d}^{(1)}-I\right\|_{H^{\beta}(\mathbb{R}) \rightarrow L^{2}(\mathbb{R})} \\
& \quad \lesssim d^{-\min \{2, \alpha\}}+d^{-\min \{2, \beta\}} .
\end{aligned}
$$

Let $f$ be in $\mathcal{C}_{0}^{\infty}(\Omega)^{3}$. Then, for $j=1,2,3$,

$$
\begin{aligned}
\left\|\left(\mathbf{E}_{d}\right)_{j} f-f_{j}\right\|_{L^{2}(\Omega)} & \leq\left\|\left(\mathbf{E}_{d}\right)_{j} f-f_{j}\right\|_{L^{2}\left(\mathbb{R}^{3}\right)} \\
& =\left\|\left(\mathbf{E}_{d}\right)_{j} f \circ \mathbf{Q}_{j}-f_{j} \circ \mathbf{Q}_{j}\right\|_{L^{2}\left(\mathbb{R}^{3}\right)} \\
& =\left\|\left(\mathbf{E}_{d}\right)_{j} f-f_{j} \circ \mathbf{Q}_{j}\right\|_{L^{2}\left(\mathbb{R}^{3}\right)} \\
& \stackrel{(\text { B. } 2)}{=}\left\|E_{d}^{(2)} \otimes E_{d}^{(1)}\left(f_{j} \circ \mathbf{Q}_{j}\right)-f_{j} \circ \mathbf{Q}_{j}\right\|_{L^{2}\left(\mathbb{R}^{3}\right)} \\
& \stackrel{(\text { B.3 })}{\lesssim}\left(d^{-\min \{2, \alpha\}}+d^{-\min \{2, \beta\}}\right) \| \underbrace{\| f_{j}}_{=\left\|f_{j}\right\|_{\mathcal{X}_{j}^{\alpha, \beta}} \circ \mathbf{Q}_{j} \|_{H^{\alpha}\left(\mathbb{R}^{2}\right) \widehat{\otimes} H^{\beta}(\mathbb{R})}}
\end{aligned}
$$

which is (3.12) since $\mathcal{C}_{0}^{\infty}(\Omega)$ is dense in $\mathcal{X}_{j}^{\alpha, \beta}$. Finally, (3.12) yields (3.11) by a density argument.

\section{References}

1. Aubin, J.-P.: Applied Functional Analysis. Pure \& Applied Mathematics, Wiley, New York, 2nd ed., 2000

2. Gradshteyn, I.S., Ryzhik, I.M.: Table of integrals, series, and products. Academic Press, San Diego, 1980

3. Jonas, P., Louis, A.K.: A Sobolev space analysis of linear regularization methods for ill-posed problems. J. Inv. Ill-Posed Problemss 9, 59-71 (2001)

4. Juhlin, P.: Principles of Doppler tomography. tech. rep., Centre for Mathematical Sciences, Lund Institute of Technology, SE-221 00 Lund, Sweden, 1992

5. Louis, A.K.: Approximate inverse for linear and some nonlinear problems. Inverse Problems 12, 175-190 (1996)

6. Louis, A.K.: A unified approach to regularization methods for linear ill-posed problems. Inverse Problems 15, 489-498 (1999)

7. Louis, A.K., Maaß, P.: A mollifier method for linear operator equations of the first kind. Inverse Problems 6, 427-440 (1990)

8. Louis, A.K., Schuster, Th.: A novel filter design technique in 2D computerized tomography. Inverse Problems 12, 685-696 (1996)

9. Louis, A.K., Natterer, F.: Mathematical problems in computerized tomography. Proceedings IEEE 71, 379-389 (1983) 
10. Schuster, Th.: The 3D Doppler transform: elementary properties and computation of reconstruction kernels. Inverse Problems 16, 701-723 (2000)

11. Schuster, Th.: An efficient mollifier method for three-dimensional vector tomography: convergence analysis and implementation. Inverse Problems 17, 739-766 (2001)

12. Natterer, F.: The Mathematics of Computerized Tomography. Wiley, Chichester, 1986

13. Norton, S.J.: Unique tomographic reconstruction of vector fields using boundary data. IEEE Trans. Image Proc. 1, 406-412 (1992)

14. Rieder, A.: Principles of reconstruction filter design in 2D-computerized tomography. In: Radon Transforms and Tomography, E. T. Quinto, L. Ehrenpreis, A. Faridani, F. Gonzales, and E. Grinberg, (eds)., vol. 278 of Contemporary Mathematics, AMS, Providence, RI, 2001, pp. 201-226

15. Rieder, A., Schuster, Th.: The approximate inverse in action with an application to computerized tomography. SIAM J. Numer. Anal. 37, 1909-1929 (2000)

16. Rieder, A., Schuster, Th.: The approximate inverse in action II: convergence and stability. Math. Comp. 72, 1399-1415 (2003)

17. Schumaker, L.L.: Spline Functions: Basic Theory. Pure \& Applied Mathematics, John Wiley \& Sons, New York, 1981

18. Sparr, G., Stråhlén, K.: Vector field tomography: an overview. tech. rep., Mathematical Imaging Group, Centre for Mathematical Sciences, Lund Institute of Technology, SE-221 00 Lund, Sweden, 1998. URL: www . maths . 1 th . se/matematiklth/vision/publications.html\#internal.

19. Weidmann, J.: Linear Operators in Hilbert Spaces. vol. 68 of Graduate Texts in Mathematics, Springer-Verlag, New York, 1980

20. Wloka, J.: Partial Differential Equations. Cambridge University Press, Cambridge, U.K., 1987 